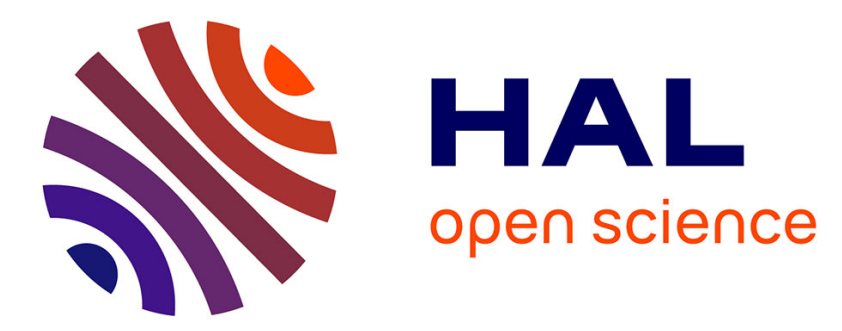

\title{
A new contact model for multi-layered solids with rough surfaces
}

Yassine Waddad, Vincent Magnier, Philippe Dufrenoy, Géry de Saxcé

\section{To cite this version:}

Yassine Waddad, Vincent Magnier, Philippe Dufrenoy, Géry de Saxcé. A new contact model for multi-layered solids with rough surfaces. Tribology Letters, 2017, 65 (4), 10.1007/s11249-017-0941-6 . hal-01737135

\section{HAL Id: hal-01737135 \\ https://hal.science/hal-01737135}

Submitted on 19 Mar 2018

HAL is a multi-disciplinary open access archive for the deposit and dissemination of scientific research documents, whether they are published or not. The documents may come from teaching and research institutions in France or abroad, or from public or private research centers.
L'archive ouverte pluridisciplinaire $\mathbf{H A L}$, est destinée au dépôt et à la diffusion de documents scientifiques de niveau recherche, publiés ou non, émanant des établissements d'enseignement et de recherche français ou étrangers, des laboratoires publics ou privés. 


\title{
A new contact model for multi-layered solids with rough surfaces
}

\author{
Yassine Waddad · Vincent Magnier · \\ Philippe Dufr'enoy · G'ery de Saxc'e
}

Received: date / Accepted: date

\begin{abstract}
A new numerical model is proposed to investigate the normal contact of multi-layered solids with rough surfaces. The Hankel transform and the transfer matrix technique are used to solve the problem of the deformation of a multi-layered solid. Then, the normal contact of an asperity is solved with Abel transform. Using this solution, an asperity-based contact model of rough surfaces is developed considering interactions between asperities. Numerical results are presented and compared to finite element calculations. The present model provides good results. The effects of interactions and the solid layers properties are discussed.
\end{abstract}

Keywords Contact analysis $\cdot$ surface roughness $\cdot$ multi-layered solid $\cdot$ Hankel transform $\cdot$ transfer matrix technique.

\section{Introduction}

Contact mechanics is of high interest in many engineering systems. For many of them, if two solids are in contact the real contact area is much smaller than the apparent one due to surface roughness. The interface behavior and the

\author{
Yassine Waddad \\ Universite of Lille-LML, F-59655 Villeneuve dAscq, France \\ E-mail: waddad.yassine.11@gmail.com \\ Vincent Magnier \\ Universite of Lille-LML, F-59655 Villeneuve dAscq, France E-mail: \\ vincent.magnier@polytech-lille.fr \\ Philippe Dufr'enoy \\ Universite of Lille-LML, F-59655 Villeneuve dAscq, France E-mail: \\ philippe.dufrenoy@polytech-lille.fr
}


G'ery de Saxc'e

Universite of Lille-LML, F-59655 Villeneuve dAscq, France

E-mail: gery.desaxce@univ-lille1.fr

contact area has a great effect on system performance [1].

Naturally, the contact interface behavior depends on the roughness of the contacting solids and their mechanical properties. A contact model considering surface roughness goes first through the description of surface roughness. For this purpose, the random process theory is commonly used to compute some relevant statistical parameters $[2,3]$ and fractal techniques are often used to characterize the multi-scale nature of rough surfaces [4,5]. For this purpose, statistical parameters are commonly used to characterize the surface [2,3] and fractal techniques are often used to describe the multi-scale nature of rough surfaces $[4,5]$.

There is a wide range of normal contact theories with rough surfaces. The classical contact theories are based on the concept of asperity which was first introduced by [6]. The classical contact theories are based on the concept of asperity introduced first in Hertz theory and used by Archard [6] to model multiscale rough surfaces. The basic idea of these theories is that contact occurs only on the top of these asperities. The well-known theory of Greenwood and Williamson [7] represents the basis of all the asperity-based models. This theory assumes that roughness can be described as a spatial distribution of spherical asperities having the same curvature radius and a randomly distributed heights. The asperities deform according to Hertz theory. An improvement of this theory has been proposed by Bush et al.[8]. They consider that each asperity is approximated by a paraboloid having the same geometric parameters as the asperity. The basic idea is to consider a random process of asperity heights and curvatures. The basic idea is to consider a random distribution of asperity heights and curvatures.

These models have achieved many results of great interest and have been successful for many years. However there is some weaknesses arising from the dependency on the statistical parameters and the non-consideration of interactions between asperities. Nevertheless, many improvements have been proposed by different authors. For instance, interactions have been included using semi-analytic approaches $[9,10,11]$. Considering interactions is in particular important for the cases close to full contact situation.

The contact problem can also be solved by means of numerical methods. Using the finite element method, the problem is solved with optimization techniques and the region near to contact surfaces has to be finely meshed to guarantee a good accuracy $[12,13]$. In return, the method is very costly in terms of CPU time which might be restrictive.

Another method is to consider the solid as a half space and solve the problem using the so-called influence coefficients $[14,15]$. Following this approach, only the surface is discretized which saves considerably CPU time. Using the Fast Fourier Transform (FFT), a fast version of this method, has been developed in [16].

The various existing models are focused on the contact surface geometry and consider that the solid is elastic and homogeneous, which is obviously not 
always true. Solids could be heterogeneous and could undergo several transformations under thermal and mechanical loadings and notably the subsurface material is the most exposed to these loadings. Hence, the bulk material properties change constantly and could affect the contact interface behavior. In several works, the material properties effect have been investigated. The plastic contact have been analyzed in [17] using the influence matrix coefficients, in [18] with the finite element method and in [19] with an improved statistical asperity model. Material heterogeneities have been considered in [20] to model fretting problems.

In many problems, the material beneath the surface can be considered as multilayered, such as coating and composites. Moreover, in braking applications and hot forging tools, there is material transformations leading to the appearance of a gradient of material properties near to contact interfaces. Thus, these properties vary in the direction normal to the contact surface. Consequently, in some way, the material can be considered as a multi-layered system where each layer is homogeneous and perfect continuity is assumed at the interface layers.

To the best of our knowledge, the contact problem involving rough surfaces with multi-layered solids has so far received a few attention. The contact between an ellipsoid and a layered half space has been studied numerically in [21]. In [22, 23], a Green function approach has been used to analyze a 2D sliding contact problem including friction. In [30, 29], Papkovich-Neuber potentials with a Fast Fourier Transform scheme (FFT), have been used to study the rough contact problem of elastic and plastic solids. These studies were carried on solids having a maximum of three layers. The problem is solved using the influence matrix method of which the coefficients are obtained by solving a linear system of equations. This system is obtained from the continuity conditions at the interface layers. Under such an approach, it is very complicated to extend the technique to cover the general case of multi-layers.

The contact problem involving rough surfaces with multi-layered solids (3 layers and more) has so far received a few attention. The contact between an ellipsoid and a layered half space has been studied numerically in [21]. In [22, 23], a Green function approach has been used to analyze a 2D sliding contact problem including friction. [24] developed a modified version of Hertz theory for coated solids and have obtained fitted curves of contact area based on the work of [25] who calculate influence coefficients of coated half spaces using Fourier transform [26]. Another deterministic technique has been proposed by [27] to obtain a closed form solution for an asperity using a perturbation technique developed by [28]. In [29, 30], Papkovich-Neuber potentials with a Fast Fourier Transform scheme (FFT), have been used to study the rough contact problem of elastic and plastic solids. The problem is solved using the influence matrix method of which the coefficients are obtained by solving a linear system of equations. In most of these works, the analyses were carried on solids having a maximum of three layers. Under such approaches, it is very complicated to extend the different methods to cover the general case of multilayers. Alternatively, the finite element method has also been used to study subsurface stresses and deformations within a flat layered medium in contact 
with a rigid sphere [31] and for the case of a rough surface described by Weierstrass fractal function[32]. More recently, with the same method, the contact of coated solids has been investigated by [33] and [34] with the objective of identifying the location of plastic yielding onset in coated solids. Although, this method can give a full description of a multi-layered structure, its numerical cost remains very considerable comparing to semi-analytic and analytic methods.

An interesting approach has been proposed by [37] who has investigated the contact problem of a multi-layered solid submitted to the indentation of a rigid circular plate using the transfer matrix technique $([35,36])$ and both the Fourier transform and Hankel transform properties [38] to obtain a Fredholm integral equation which is solved to obtain a closed-form solution for the contact problem. The main advantage of this technique is the ability to relate directly surface stresses to surface displacements which is very useful in solving contact and surface loading problems.

For multi-layered solids (3 layers and more), an interesting approach seems to be more convenient. It consists of defining a transfer matrix for each solid layer and then relating surface stresses to surface displacements with a transfer function depending on these matrices $[35,36]$. This technique has been used by [37] to calculate the stresses in a multi-layered solid submitted to the indentation of a rigid circular plate. To do that, He used a technique developed by [38] and have obtained a Fredholm integral equation which is solved to obtain a closed-form solution for the contact problem as in $[39,40]$. Recently, the same technique has been used by [41] to solve the axisymmetric indentation problem of multi-layered solids.

In this paper, a rough contact model of multi-layered solids is proposed based on the transfer matrix technique [36] and the works of [39, 37]. First, by making use of the transfer matrix technique, the surface displacements are expressed in terms of surface stresses for a multi-layer problem. Second, a contact model of an axially symmetric asperity is developed by extending the solutions proposed by $[39,37]$. Then a closed form solution is obtained for the same problem. Subsequently, by considering the classical multi-asperity surface description, the contact asperity model is used to solve the rough contact problem including interactions between asperities. Finally, typical results are presented and compared to Finite Element calculations. In particular, the effect of the solid layers elastic properties and the asperities interactions are discussed. Moreover, several calculations are performed on many surface samples with the aim to highlight the effect of the surface spectrum breadth.

\section{Surface loading of multi-layered elastic solid : the transfer matrix technique}

2.1 General framework 
Considering an isotropic elastic medium $(z \geq 0)$ laterally unbounded (see Fig.1). There is no body forces, the static equilibrium of the solid can be written as follows

$$
\sigma_{i j, j}=0
$$

where $\sigma$ is the Cauchy stress tensor and $x_{j}$ is the $1^{\text {st }}$ order partial derivative of a given operand $x$ per the $j^{t h}$ component.

The deformation in the solid is assumed to be infinitesimal, thus, the strains $i j$ are related to the displacements $u_{i}$ by

$$
\epsilon_{i j}=\frac{1}{2}\left(u_{i, j}+u_{j, i}\right)
$$

The constitutive based material has elastic properties varying with depth $z$. Thus, one can consider the solid as a multi-layered system of unbounded horizontal layers, where each layer has its own local properties (see Fig.1).

For the $k^{\text {th }}$ layer, the constitutive equation between stresses $\sigma_{i j}$ and strains ${ }_{i j}$ is expressed by Hooke's law

$$
\sigma_{i j}=2 \mu_{k}\left(\varepsilon_{i j}+\frac{\nu_{k}}{1-2 \nu_{k}} \varepsilon_{q q} \delta_{i j}\right)
$$

where $\mu_{k}$ is the shear modulus, $v_{k}$ is the Poisson's ratio and $\delta_{i j}$ is the Kronecker symbol.

Solving the contact problem is to express the displacements and the stresses in the contacting regions of the top surface of the solid $(z=0)$. For that issue, the problem is solved through the Hankel integral transform and the transfer matrix technique.

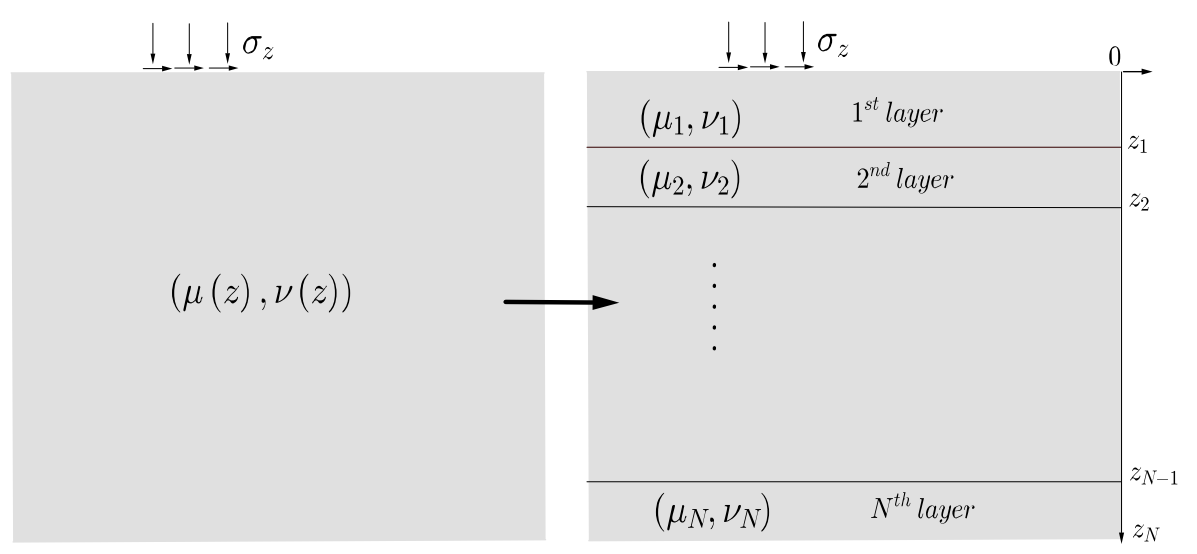

Fig. 1 Schematic of a multi-layered elastic solid under surface loads 2.2 The transfer matrix technique 
Let us consider the axis of symmetry of an isotropic elastic medium as the $z$-axis. The governing equations are presented as follows in cylindrical coordinates $(r, \theta, z)$. The problem is solved in the case of axially symmetric deformation and all field variables are independent of $\theta$.

Considering the stress vector defined by $\mathbf{T}_{z}=\left[\sigma_{r z}, \sigma_{\theta z}, \sigma_{z z}\right]^{t}$ and the displacements vector $\mathbf{u}=\left[u_{r}, u_{\theta}, u_{z}\right]^{t}$. Let us introduce the following set of solution representation using the Hankel integral transform

$$
\begin{aligned}
& =\left[\begin{array}{l}
w_{\xi} \\
w_{\eta} \\
w_{z}
\end{array}\right]=\int_{0}^{+\infty}\left[\begin{array}{ccc}
-J_{1}(\rho r) / \rho & 0 & 0 \\
0 & J_{1}(\rho r) / \rho & 0 \\
0 & 0 & J_{0}(\rho r)
\end{array}\right] \cdot \mathbf{u} r \mathrm{~d} r \\
\boldsymbol{\tau} & =\left[\begin{array}{c}
\tau_{\xi} \\
\tau_{\eta} \\
\tau_{z}
\end{array}\right]=\int_{0}^{+\infty}\left[\begin{array}{ccc}
-J_{1}(\rho r) / \rho & 0 & 0 \\
0 & J_{1}(\rho r) / \rho & 0 \\
0 & 0 & J_{0}(\rho r)
\end{array}\right] . \mathbf{T}_{z} r \mathrm{~d} r
\end{aligned}
$$

where $J_{n}$ is the $n^{\text {th }}$ order Bessel function.

It is well known that this set of variables satisfies the two decoupled first order differential equations

$$
\begin{aligned}
& -\left[\begin{array}{c}
w_{\eta} \\
-
\end{array}\right]=\left\lceil\begin{array}{ll}
0 & -1
\end{array}\left[\begin{array}{l}
w_{\eta} \\
\end{array}\right]\right. \\
& \text { d }
\end{aligned}
$$

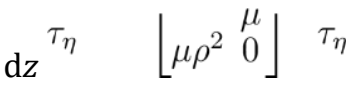

$$
\begin{aligned}
& {\left[\begin{array}{c}
w_{z} \\
\rho w_{\xi}
\end{array}\right\rceil=\left[\begin{array}{cccc}
0 & \frac{\nu \rho}{1-\nu} & \frac{(1-2 \nu) \rho}{2 \mu(1-\nu)} & 0 \\
-\rho & 0 & 0 & \underline{\rho}
\end{array}\right\rceil\left\lceil\begin{array}{c}
w_{z} \\
\rho w_{\xi}
\end{array}\right\rceil}
\end{aligned}
$$

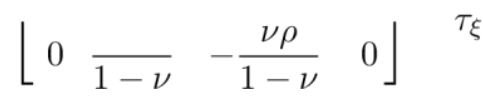

$$
\begin{aligned}
& \text { d } \\
& \mathrm{d}\left\lfloor\left\lfloor\begin{array}{c}
\tau_{z} / \rho \\
\tau_{\xi}
\end{array}\right\rfloor \quad\left|\begin{array}{cccc}
0 & 0 & 0 & \rho \\
& 2 \mu \rho & &
\end{array}\right| \tau_{z} / \rho\right\rfloor
\end{aligned}
$$

The global solution of this system is an eigenvalue extraction problem and can be written in the following form

$$
\left[\begin{array}{c}
w_{\eta} \\
\tau_{\eta}
\end{array}\right]=\mathbf{Z}_{1}\left[\begin{array}{l}
A_{\eta} \\
B_{\eta}
\end{array}\right] \text { and }\left[\begin{array}{c}
w_{z} \\
\rho w_{\xi} \\
\tau_{z} / \rho \\
\tau_{\xi}
\end{array}\right]=\mathbf{Z}_{2}\left[\begin{array}{c}
A_{z} \\
A_{\xi} \\
B_{z} \\
B_{\xi}
\end{array}\right]
$$

where $A_{z}, A_{\eta}, B_{\eta}, B_{Z}, A_{\xi}$ and $B_{\xi}$ are constants and the matrices $\mathbf{Z}_{1}$ and $\mathbf{Z}_{2}$ are given in Appendix A.

Considering that the medium is an unbounded horizontal layer of which the thickness is $h$ (see Fig.2). The superscript ${ }^{(+)}$(resp. ${ }^{(-)}$) is used for the top layer surface variables (resp. the bottom one). One can show that 


$$
\left[\begin{array}{c}
w_{\eta}^{(+)} \\
\tau_{\eta}^{(+)} / \rho
\end{array}\right]=\mathbf{T}_{\eta}\left[\begin{array}{c}
w_{\eta}^{(-)} \\
\tau_{\eta}^{(-)} / \rho
\end{array}\right] \text { and }\left[\begin{array}{c}
w_{z}^{(+)} \\
\rho w_{\xi}^{(+)} \\
\tau_{z}^{(+)} / \rho \\
\tau_{\xi}^{(+)}
\end{array}\right]=\mathbf{T}_{z \xi}\left[\begin{array}{c}
w_{z}^{(-)} \\
\rho w_{\xi}^{(-)} \\
\tau_{z}^{(-)} / \rho \\
\tau_{\xi}^{(-)}
\end{array}\right]
$$

where $\mathbf{T}_{z \xi}$ and $\mathbf{T}_{\eta}$ are the transfer matrices of the layer and are given in Appendix A.

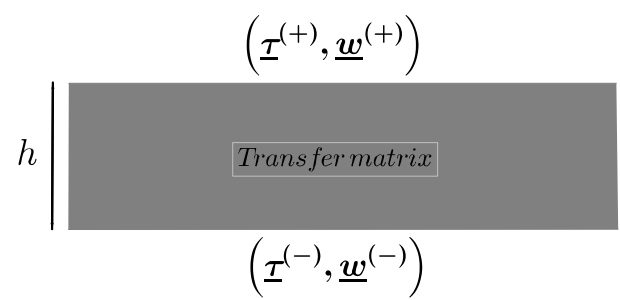

Fig. 2 Schematic of an elastic layer of which the deformations and the stresses are given by the transfer matrix technique

Now let us consider a multi-layered body made of $N$ horizontal layers Fig.1. The $1^{s t}$ layer is on the top of the solid and the $N^{t h}$ layer lies on a homogeneous half space that can be either rigid or elastic. Considering the perfect continuity between the parallel layers, then the displacement field $\mathbf{u}$ and the stress vector $\mathbf{T}_{z}$ are both continuous. Using the transfer matrices, one can obtain the following set of equations

$$
\begin{aligned}
& \text { 回 } w_{z}(0) \text { 目目 } w_{z}(N) \text { ? }
\end{aligned}
$$

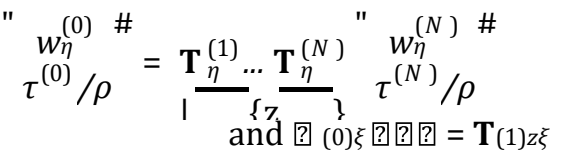

$$
\begin{aligned}
& \text { (0) } \\
& \tau_{\xi}^{(0)} \text { ता }-\stackrel{\text { ? } \rho w}{=} \text { ? } \tau_{\xi}^{(N)} \text { ? }
\end{aligned}
$$

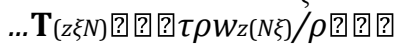

$$
\begin{aligned}
& \text { ? } \\
& \eta \quad \tau_{z} / \rho \\
& \mid\{\mathrm{z}\}
\end{aligned}
$$

From these equations, it can be seen that the transformed stresses and displacements of the top surface of the $1^{\text {st }}$ layer can be related directly to those of the bottom surface of the $N^{t h}$ layer by the mean of the matrices $\mathbf{S}$ and $\mathbf{R}$. These matrices are given by a simple product of the transfer matrices of the intermediate layers as shown in these equations.

In order to solve the problem, the boundary conditions in the bottom face are used. Following many algebraic operations, one can show that 


$$
\left[\begin{array}{c}
w_{z}^{(0)} \\
\rho w_{\xi}^{(0)} \\
w_{\eta}^{(0)}
\end{array}\right]=\mathbf{F}\left[\begin{array}{c}
\tau_{z}^{(0)} / \rho \\
\tau_{\xi}^{(0)} \\
\tau_{\eta}^{(0)} / \rho
\end{array}\right], \text { where } \mathbf{F}=\left[\begin{array}{ccc}
F_{11} & F_{12} & 0 \\
F_{21} & F_{22} & 0 \\
0 & 0 & F_{33}
\end{array}\right]
$$

The matrix $\mathbf{F}$ is called the transfer matrix of the body. The matrix coefficients depend on the bottom surface boundary conditions and are given in Appendix B. In Eq.5, one can see that surface displacements are related directly to surface loads using the matrix F. More clearly, if a general surface loading is applied to the top surface $z=0$, one can compute the real displacements automatically using the inverse Hankel transform of Eq.5.

At last, it has to be noticed that the transfer matrix can also be formulated in Cartesian coordinates with the use of Fourier integral transform.

In what follows, the normal elastic contact problem of an axially symmetric asperity is solved by making use of the transfer matrix and integral transforms.

\section{Elastic contact of an axially symmetric asperity}

\subsection{General contact problem}

Considering a multi-layered solid, the top surface shape of the solid contains an asperity which is described by a function $z=f(r)$. The small-slope approximation is considered. The asperity is compressed normally against a rigid plan with a vertical motion $\delta$. Just as in Hertz theory, a circular contact zone appears and has a radius $a$. Also, no shear stresses are considered. The boundary conditions of the problem are :

- Inside the contact: $u_{z}(r)=\delta-f(r), 0 \leq r \leq a$

- Outside the contact: $\sigma_{z z}(r)=0, a \leq r$

As shown in the previous section, using the transfer matrix technique, one can show that

$$
w_{z}=F_{11}(\rho)\left(\tau_{z} / \rho\right)
$$

Using inverse Hankel transform, the boundary conditions can be written as follows

- Inside contact:

$$
u_{z}(r)=\delta-f(r)=\int_{0}^{+\infty} F_{11}(\rho) \tau_{z}(\rho) J_{0}(\rho r) \mathrm{d} \rho, 0 \leq r \leq a
$$

- Outside contact:

$$
\int_{0}^{+\infty} \tau_{z}(\rho) J_{0}(\rho r) \rho \mathrm{d} \rho=0, a \leq r
$$

Let us denote 


$$
\alpha=\lim _{\rho \rightarrow \infty} \frac{1}{F_{11}(\rho)}
$$

One can show that for any given boundary conditions, we have

$$
\alpha=\frac{\mu_{1}}{\nu_{1}-1}
$$

where $\mu_{1}$ et $v_{1}$ are the elastic parameters of the first layer. Consequently, we introduce the kernel $k_{z}$ defined by

$$
k_{z}(\rho)=\alpha F_{11}(\rho)-1
$$

Then Eq.(6) becomes

$\alpha(\delta-f(r))=\begin{gathered}\mathrm{Z}+\infty \\ 0\end{gathered} \tau_{z}(\rho) J_{0}(\rho r) \mathrm{d} \rho+{ }_{0}^{\mathrm{Z}+\infty} \quad k_{z}(\rho) \tau_{z}(\rho) J_{0}(\rho r) \mathrm{d} \rho, 0 \leq r \leq a$

In order to solve this singular integral equation, we will refer to the earlier works of $[39,37]$. Therefore, the auxiliary function $\varphi$ is introduced by

$$
\tau_{z}(\rho)=\int_{0}^{a} \phi(x) \cos (\rho x) \mathrm{d} x
$$

We also introduce the Abel transform A defined by

$$
\mathcal{A}(f(x), r)=\frac{2}{\pi} \int_{0}^{r} \frac{f(x)}{\sqrt{r^{2}-x^{2}}} \mathrm{~d} x
$$

Knowing that

$$
J_{0}(\rho r)=\mathrm{A}(\cos (\rho x), r)
$$

then

$$
\int_{0}^{+\infty} \tau_{z}(\rho) J_{0}(\rho r) \mathrm{d} \rho=\mathcal{A}\left(\frac{\pi}{2} \phi(x), r\right)
$$

and

$\int_{0}^{+\infty} k_{z}(\rho) \tau_{z}(\rho) J_{0}(\rho r) \mathrm{d} \rho=\mathcal{A}\left(\int_{0}^{+\infty} \int_{0}^{a} k_{z}(\rho) \phi(x) \cos (\rho x) \cos (\rho r) \mathrm{d} x \mathrm{~d} \rho, r\right)$

Hence Eq. (7) may be written as an Abel integral equation

$$
\mathcal{A}\left(\frac{\pi}{2} \phi(x)+\int_{0}^{+\infty} \int_{0}^{a} k_{z}(\rho) \phi(x) \cos (\rho x) \cos (\rho r) \mathrm{d} x \mathrm{~d} \rho, r\right)=\alpha(\delta-f(r))
$$

Making use of the inverse Abel transform $\mathrm{A}^{-1}$ and the integration by parts, one can obtain

$$
\frac{\pi}{2} \phi(r)+\int_{0}^{+\infty} \int_{0}^{a} k_{z}(\rho) \phi(x) \cos (\rho x) \cos (\rho r) \mathrm{d} x \mathrm{~d} \rho=\alpha\left(\delta-f(0)-\int_{0}^{r} \frac{r f^{\prime}(x)}{\sqrt{r^{2}-x^{2}}} \mathrm{~d} x\right)
$$

Using Abel integral transform [38, 37], one can obtain from Eq.(7)

$$
\frac{\pi}{2} \phi(r)+\int_{0}^{+\infty} \int_{0}^{a} k_{z}(\rho) \phi(x) \cos (\rho x) \cos (\rho r) \mathrm{d} x \mathrm{~d} \rho=\alpha\left(\delta-f(0)-\int_{0}^{r} \frac{r f^{\prime}(x)}{\sqrt{r^{2}-x^{2}}} \mathrm{~d} x\right)
$$


Introducing the dimensionless variables $s=r / a$ and $t=x / a$, a Fredholm equation of the second order is obtained

$$
\phi_{a}(s)+\int_{0}^{1} K_{a}(s, t) \phi_{a}(t) \mathrm{d} t=G(s)
$$

where

$$
K_{a}(s, t)=\frac{2}{\pi} \int_{0}^{+\infty} k_{z}(\rho / a) \cos (\rho s) \cos (\rho t) \mathrm{d} \rho
$$

and

$$
\phi_{a}(u)=\frac{\pi}{2} \phi(a . u)
$$

and

$$
\left.G(s)=\alpha\left(\delta-f(0)-a \int_{0}^{s} \frac{s f^{\prime}(a t)}{\sqrt{s^{2}-t^{2}}} \mathrm{~d} t\right)\right)
$$

Solving Eq.(9) is done numerically by a quadrature technique. Indeed, by choosing an interpolation base, the equation can be written in a matrix format

$$
\varphi+\mathbf{K} \cdot \varphi=\mathbf{G}
$$

where $\varphi, \mathbf{K}$ and $\mathbf{G}$ are respectively the discretized form of $\varphi, K_{a}$ and $G$ expressed in integration points.

Then $\varphi$ is found by simple inversion of the matrix equation -1

$$
\varphi=(\mathbf{I}+\mathbf{K}) \quad \mathbf{G}
$$

Once the auxiliary function $\varphi_{a}$ is computed, one can calculate the total normal force $P$ by

$$
P=-4 a \int_{0}^{1} \phi_{a}(t) \mathrm{d} t
$$

At last, all surface stresses and displacements can be deduced using Hankel transform properties. In particular, we have

- The normal stress

$$
\sigma_{z z}(s)=\frac{2}{\pi a}\left(\frac{\phi_{a}(1)}{\sqrt{1-s^{2}}}-\int_{s}^{1} \frac{\phi_{a}^{\prime}(t)}{\sqrt{t^{2}-s^{2}}} \mathrm{~d} t\right) \ldots s=r / a \leq 1
$$

If $f$ is smooth, contact stress vanishes at the edge of contact zone $(r=a)$, hence, it comes that $\varphi_{a}(1)=0$ and

$$
\sigma_{z z}(s)=-\frac{2}{\pi a} \int_{s}^{1} \frac{\phi_{a}^{\prime}(t)}{\sqrt{t^{2}-s^{2}}} \mathrm{~d} t \ldots s=r / a \leq_{1}
$$

- Contact radius $a$ in the case where $f$ is smooth

$$
\begin{array}{r}
\delta=f(0)+a \int_{0}^{1} \frac{f^{\prime}(a t)}{\sqrt{\left(1-t^{2}\right)}} \mathrm{d} t+\frac{1}{\alpha} \int_{0}^{1} K_{a}(1, t) \phi_{a}(t) \mathrm{d} t \quad(12) \text { - The normal } \\
\text { displacement outside contact area } \\
u_{z}(s)=\frac{2}{\alpha \pi} \int_{0}^{1} \phi_{a}(t)\left(\frac{1}{\sqrt{s^{2}-t^{2}}}+\int_{0}^{\infty} k_{z}(\rho / a) \cos (\rho t) J_{0}(\rho s) \mathrm{d} \rho\right) \mathrm{d} t
\end{array}
$$


where $s=r / a>1$

It is important to state that the solution of [39] of elastic half spaces can be deduced by considering $k_{z}=0$, whereas the solution given by [37] can be retrieved by choosing $f=0$, which corresponds to the flat contact case. In other words, the proposed solution is an extension of both solutions to cover the axially symmetric frictionless contact problem of multi-layered solids.

3.2 Contact solution for a parabolic asperity

Considering the case of an asperity of parabolic shape. In this case, by taking the origin on the symmetry axis, the function $f$ is given by

$$
f(r)=\frac{r^{2}}{2 R}
$$

Hence the

Fredholm equation (9) $\phi_{a}(s)+\int_{0}^{1} K_{a}(s, t) \phi_{a}(t) \mathrm{d} t=\alpha\left(\delta-\frac{(a s)^{2}}{R} !\right.$

In this equation, the unknown is the contact radius $a$. Eq.(13) is solved iteratively starting from an initial trial value $\sqrt{ } a_{0}$ which can be chosen for instance

from Hertz theory $a_{0}=\quad R . \delta$.

In order to illustrate the results obtained with this model, some calculations have been performed on a single asperity model of a two-layered solid (see Fig.3(a)). The asperity is in contact with a rigid flat plane that is moved vertically against the asperity with a given displacement $\delta$.

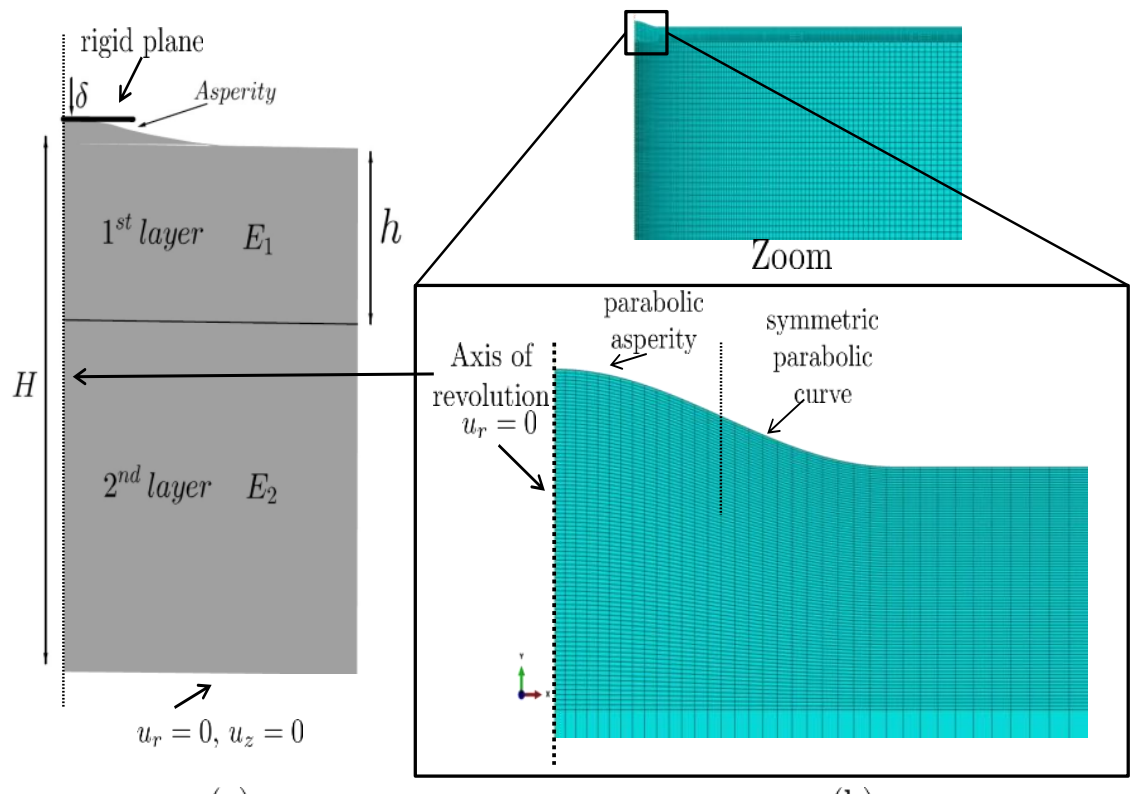

(a)

(b) 
Fig. 3 (a) Schematic of the single asperity model. The asperity is compressed vertically with a displacement $\delta$. The solid is composed of two elastic layers. (b) The finite element mesh of the

asperity model. The mesh is refined near to the top of the asperity.

The total height of the solid is $H=1 \mathrm{~mm}$ and the Young modulus of the first layer is $E_{1}=4 G P a$. Both of these quantities are fixed in this study, while the first layer thickness $h$ is varied as well as the second layer modulus $E_{2}$. The Poisson ratio $v$ $=0.15$ is the same for both layers.

The obtained results are compared to finite element calculations (FEM) and Hertz theory. The latter corresponds to the case of a homogeneous half space. The idea is to evaluate the accuracy of the model comparing to FEM calculations and to enhance the role of the sub-layers parameters.

The geometry, boundary conditions and the mesh of the FEM model are shown in Fig.3(b). The surface considered in this section is described by a parabolic asperity followed by a symmetric parabola having the same curvature. This parabola has been used to guarantee the continuity of the surface slope. Besides, this curve stands outside the contact area so that it does not affect the system's response. A quadratic hexahedron element type is used and the mesh has been refined to capture all the details of the asperity. A convergence test has been performed. For example in Fig.3(b), $17.10^{3}$ elements are used and the element size at the top of the asperity is $0.5 \mu \mathrm{m}$. Axisymmetric boundary conditions are applied at the axis of revolution of the asperity $\left(u_{r}=0\right)$ and the displacement is fixed on the bottom of the solid $\left(u_{r}=u_{z}=0\right)$. The lateral bounds are free from any boundary conditions and the width of the solid is high enough so that the layers can be considered as unbounded. Moreover, a perfect adherence is considered between the layers to guarantee the continuity of displacement and stresses. Regarding the contact interface between the rigid plane and the solid, the augmented Lagrangian method is employed. The evolution of the total force $P$ with the displacement is presented in Figs.4 -6. In Fig.4, the elastic modulus ratio $E_{2} / E_{1}$ is varied from 0.2 to 5 and the thickness $h$ is fixed at $100 \mu m$. As we can see, the predicted force deviates from Hertz theory predictions when the ratio $E_{2} / E_{1}$ varies. For the unit ratio, there is a slight difference which is due to boundary conditions effect. Moreover, comparing the model predictions with finite element results (marked by FEM in the figure and drawn by dashed lines) show a good consistency. In Fig.5, the effect of the first layer thickness is highlighted. The predicted results match FEM results. The less the thickness is, the more the results deviate from Hertz theory. Additionally, the curve plotted in Fig.6 shows the predicted values for different values of asperity radius and fixed elastic parameters. Once again, there is a good consistency between the obtained results and FEM values.

As summary, the proposed contact solution is in good accordance with FEM calculations and the force-displacement evolution is affected by the layers properties and thicknesses. In the following, the contact solution is simplified and used to model the normal contact of rough surfaces considering solid layers. 


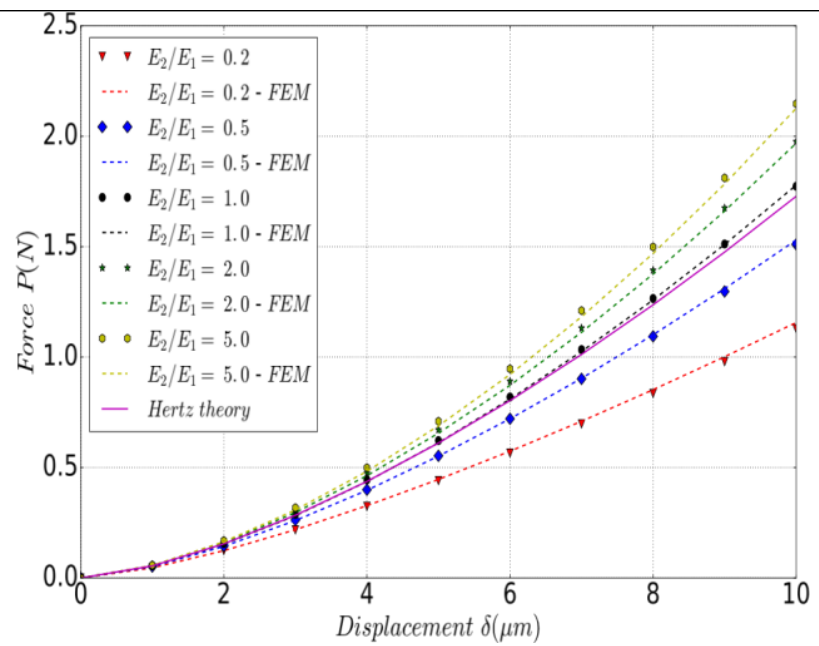

Fig. 4 Variation of the total force with the vertical displacement for the parameters : $h=100 \mu \mathrm{m}$ and $R=100 \mu m$

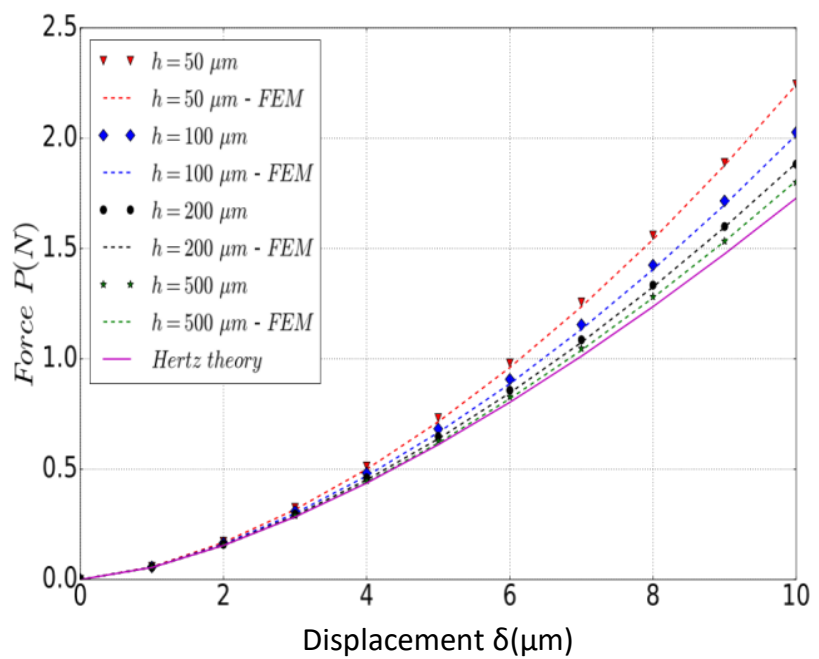

Fig. 5 Variation of the total force with the vertical displacement for the parameters : $E_{2}=10 \mathrm{GPa}$ and $R=100 \mu \mathrm{m}$

\section{Contact of rough surfaces}

4.1 An approximate asperity contact model

In the previous section, a contact model of asperities has been presented. In order to make use of this model, some simplifications are proposed. First, let us 


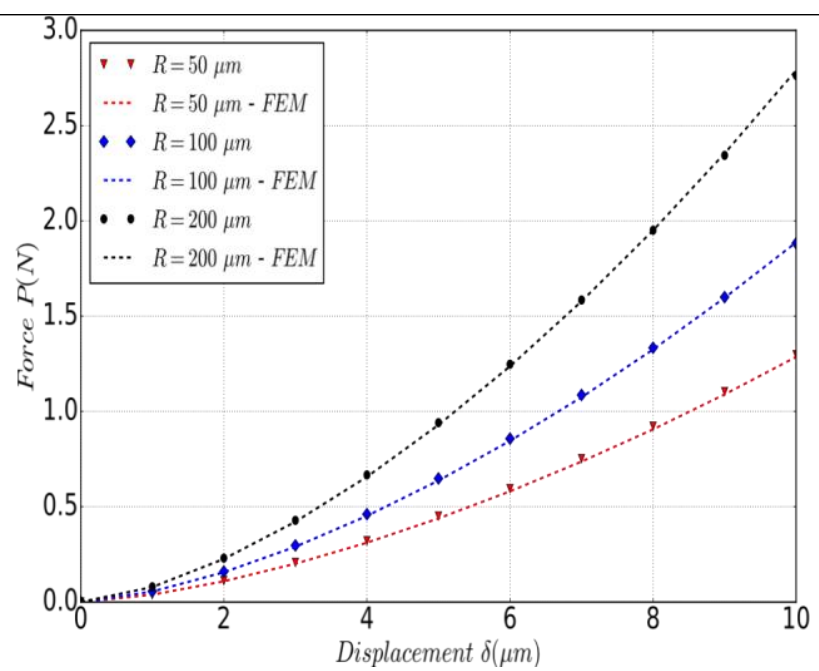

Fig. 6 Variation of the total force with the vertical displacement for the parameters : $E_{2}=10 G P a$ and $h$ $=100 \mu \mathrm{m}$

consider a parabolic asperity. The derivation of the Fredholm integral equation Eq.9 gives

$$
\overline{\phi_{a}}(s)+\int_{0}^{1} \overline{\partial K_{a}}(s, t) \phi_{a}(t) \mathrm{d} t=-\alpha \overline{2 a^{2} s}
$$

$\partial K_{a} \quad \mathrm{~d} \varphi_{a}$

$$
\text { Since } \frac{}{\partial s}(0, t)=0 \text {, one can deduce that } \frac{}{\mathrm{d} s}(0)=0
$$

Hence, by considering that the function $\varphi_{a}$ is a $2^{\text {nd }}$ order polynomial, one can write

$$
\phi_{a}(s)=\phi_{a}(0)\left(1-s^{2}\right)
$$

Using this simplified form and based on Eq.(9) and Eq.(12), $\varphi_{a}(0)$ and $\delta$ are respectively given by

$$
\begin{gathered}
\phi_{a}(0)=\frac{\alpha \delta}{1+\int_{0}^{1} K_{a}(0, t)\left(1-t^{2}\right) \mathrm{d} t} \\
\delta=\frac{a^{2}}{R}+\frac{\phi_{a}(0)}{\alpha} \int_{0}^{1}\left(1-t^{2}\right) K_{a}(1, t) \mathrm{d} t
\end{gathered}
$$

Making use of the two last equations, one can write

Using this simplified form, one can write from Eq.(9) and Eq.(12) 


$$
\phi_{a}(0)=\frac{\alpha a^{2}}{R} \chi(a) \text { and } \delta=\frac{a^{2}}{R} \zeta(a)
$$

where $\zeta$ and $\chi$ are functions of $a$

$$
\begin{gathered}
\chi(a)=\frac{1}{1+\int_{0}^{1}\left(1-t^{2}\right)\left(K_{a}(0, t)-K_{a}(1, t)\right) \mathrm{d} t} \\
\zeta(a)=\left(1+\int_{0}^{1}\left(1-t^{2}\right) K_{a}(0, t) \mathrm{d} t\right) \chi(a)
\end{gathered}
$$

Finally, the following approximated expressions are deduced:

- The total normal force $P$

$$
P=-\frac{8}{3} \alpha \chi(a) \frac{a^{3}}{R}
$$

- The normal stress $\sigma_{z z}$

$$
\sigma_{z z}(r)=\frac{4 \alpha}{\pi R} \chi(a) \sqrt{a^{2}-r^{2}} \text { if } r \leq a
$$

- The normal displacement is then given by

$$
\begin{aligned}
& u(r)=\left\{\begin{array}{c}
\frac{1}{2 R}\left(2 a^{2} \zeta(a)-r^{2}\right) \\
(
\end{array}\right. \\
& \text { ( } \frac{\chi(a)}{\pi R}\left[\left(2 a^{2}-r^{2}\right) \arcsin \left(\frac{a}{r}\right)+a \sqrt{r^{2}-a^{2}}+g(a, r)\right] \text { else. } \\
& \text { where } g(a, r)=4 \int_{0}^{\infty} k_{z}(\rho) J_{0}(\rho r) \frac{\sin (a \rho)-a \rho \cos (a \rho)}{\rho^{3}} \mathrm{~d} \rho
\end{aligned}
$$

By using this simplified form By using the parameters $\chi$ and $\zeta$, one can express all the variables as a function of the contact radius $a$, which is very helpful for the case of rough surfaces as it will be presented in the next section. In particular, the normal force and the stress and the displacement are given in Appendix C. But before tackling the problem of rough surfaces, the parameters $\chi$ and $\zeta$ are plotted in Figs.7-10 for the same configurations studied in the previous section. For small values of contact radius $a$, these parameters are close to 1 . In this case, the predicted values of the total force is almost the same as those predicted by Hertz theory.

Increased ratio $E_{2} / E_{1}$ and or decreased $h$ both lead to too much small values of $\zeta$ corresponding to too much large contact radius for a given displacement, and to too much large values of $\chi$ resulting in more important force values. Conversely, reducing $E_{2} / E_{1}$ and or raising $h$ lead to contact area and strength lowering. These parameters can be seen, in a certain manner, as sub-layer impact coefficients. Indeed, if the sub-layers have elastic parameters that are very different from those of the first one or if the first layer is very thin, these coefficients will influence strongly the contact behavior, which is the case in the 
predicted curves shown in Figs.4-6. As stated before, this approximate solution is ob-

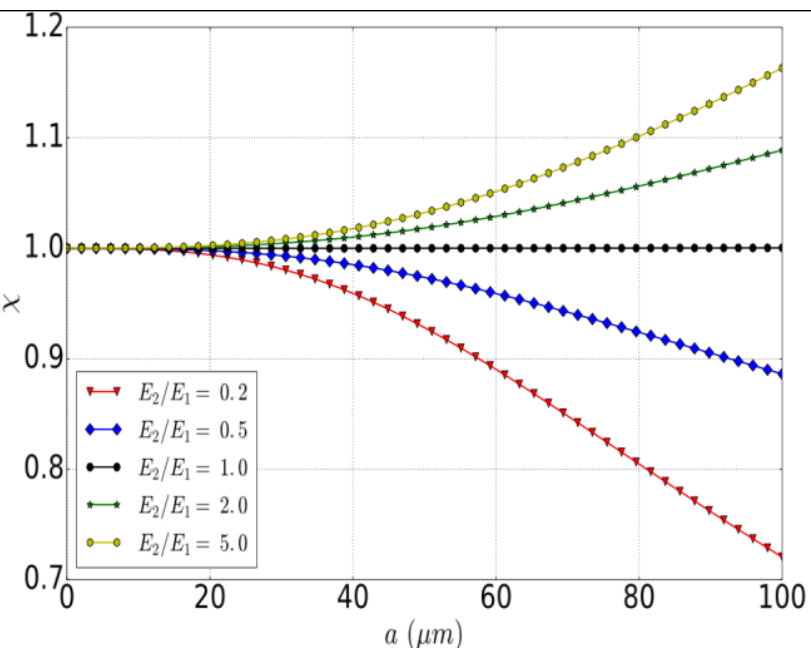

Fig. 7 Evolution of function $\chi$ for the case of $h=100 \mu \mathrm{m}$

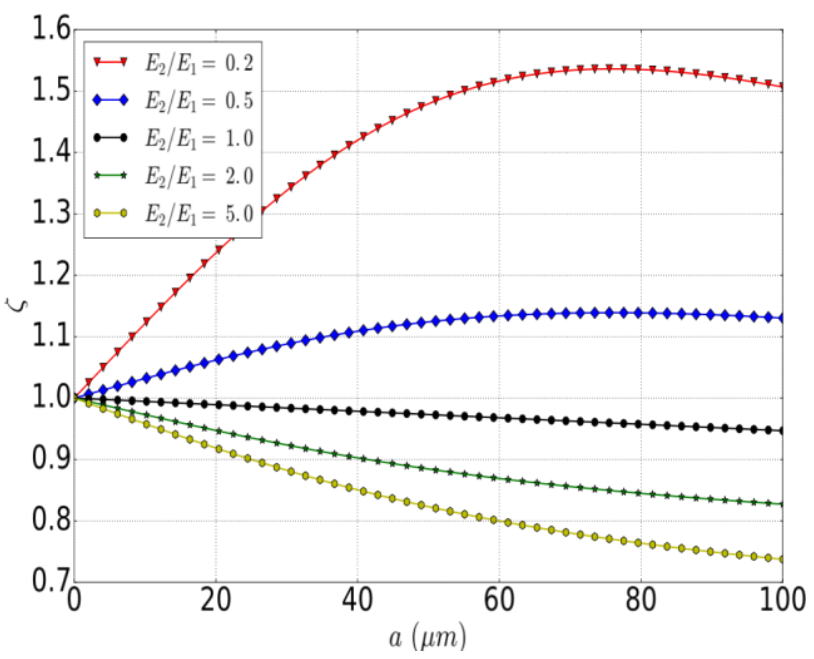

Fig. 8 Evolution of function $\zeta$ for the case of $h=100 \mu \mathrm{m}$

tained using a $2^{\text {nd }}$ order approximation of the auxiliary function. In Figs.1112, the relative error between the total force predicted using the approximate solution and the one issued from the theory is drawn for the same tested configurations. As we can see, the maximum relative error does not exceed $1 \%$ except for the case of the thinnest layer $(h=50 \mu \mathrm{m})$ where the value of $4 \%$ is reached, which is still acceptable. 


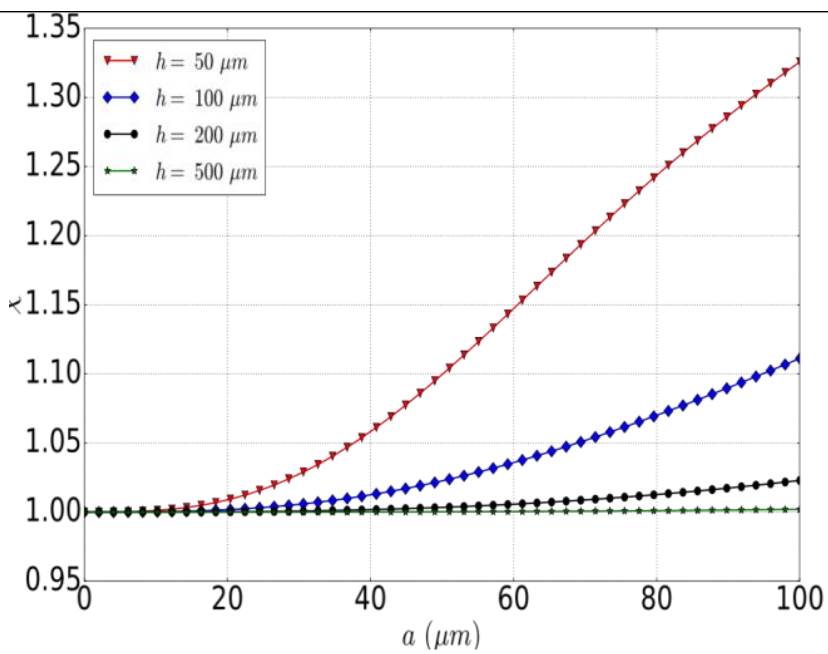

Fig. 9 Evolution of function $\chi$ for the case $E_{2}=10 \mathrm{GPa}$

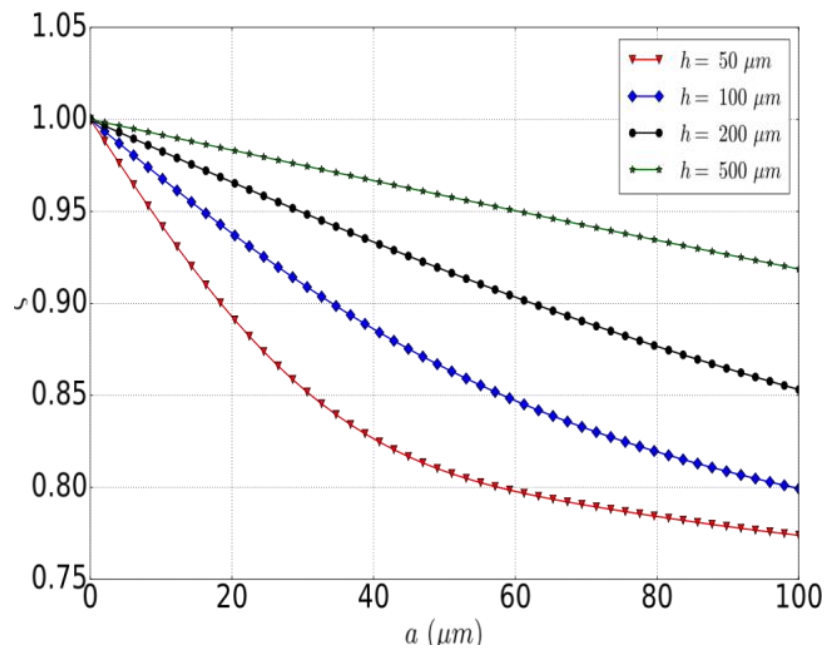

Fig. 10 Evolution of function $\zeta$ for the case $E_{2}=10 \mathrm{GPa}$

The approximation introduced in this paragraph is of great importance because it significantly simplifies the asperity contact model. Using this approximation a multi-asperity contact model is presented in the following section. 


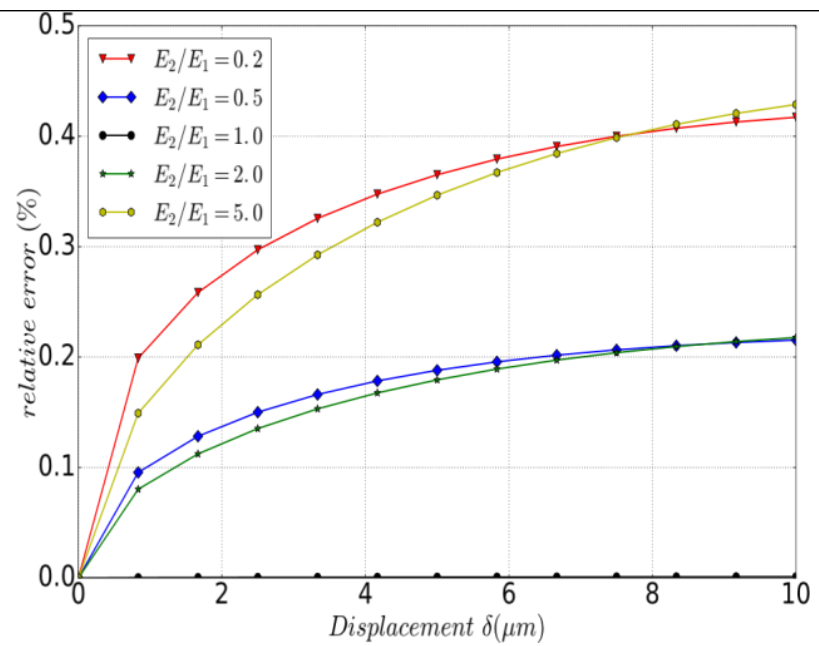

Fig. 11 The relative error between the theoretical predictions of the total force and those of the approximated model for $h=100 \mu \mathrm{m}, R=100 \mu \mathrm{m}$

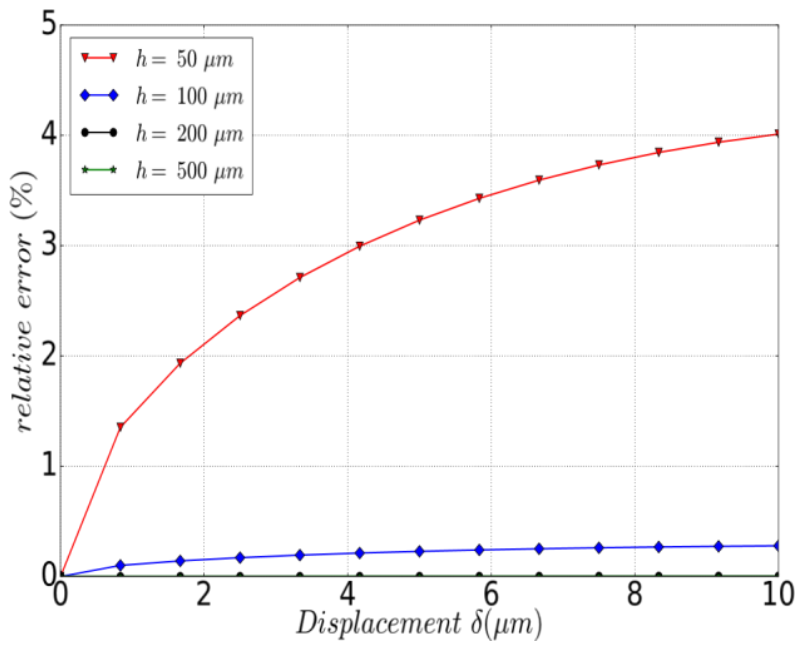

Fig. 12 The relative error between the theoretical predictions of the total force and those of the approximated model for $E_{2}=10 G P a, R=100 \mu m$

\subsection{A multi-asperity contact model}

In this section, we are dealing with the contact problem between a rough surface and a flat one. The following geometric description is similar to the ones given in $[9,11]$. The body containing the rough surface is elastic and is considered as 
a multi-layered solid. The flat surface is rigid and is moved normally with a displacement $\delta$ with respect to the rough surface. Within the frame-

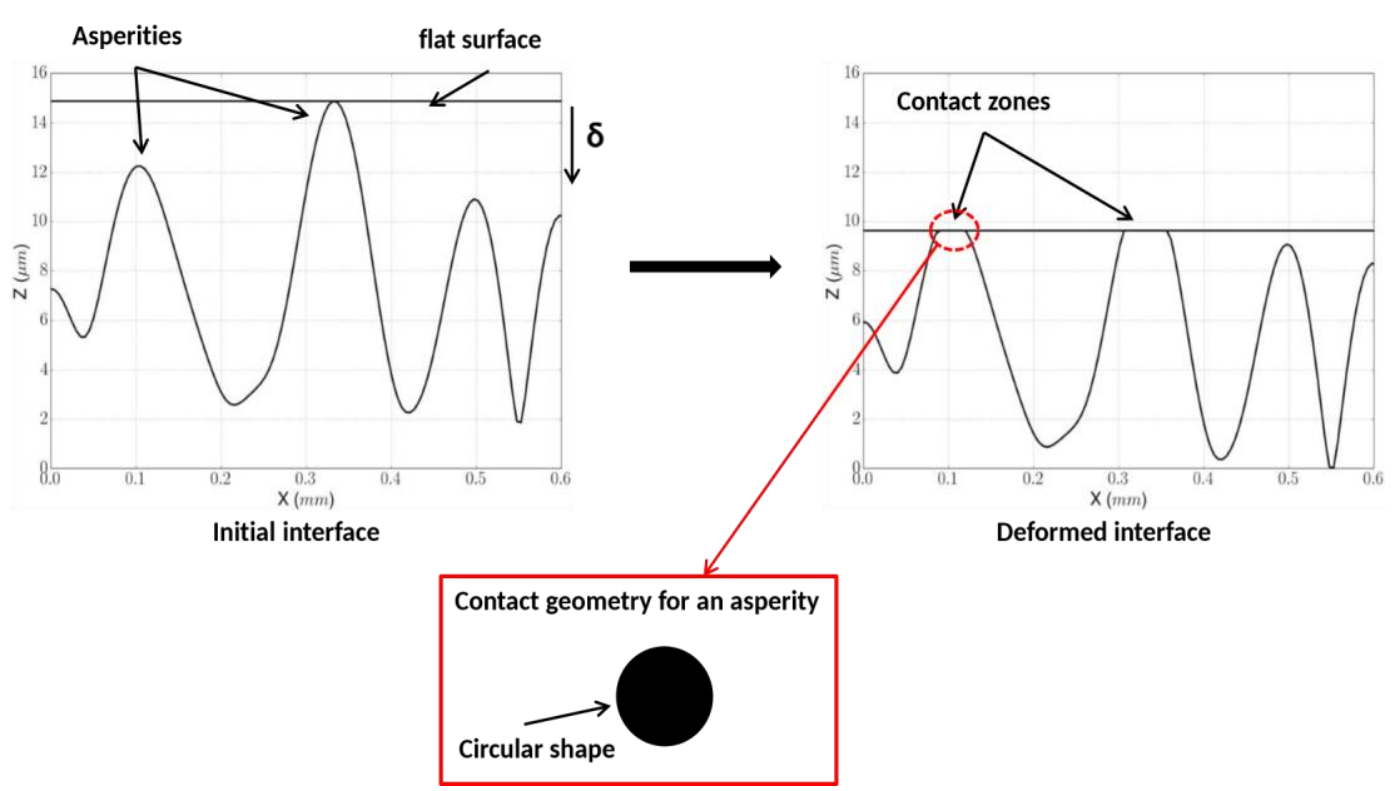

Fig. 13 Geometry of rough contact problem within a multi-asperity approach. Contact occurs only on asperities and the contact area shape in an asperity is circular.

work of a multi-asperity approach, the normal displacement in every point is a consequence of all the contact strengths forces applied on surface asperities. Taking into account the interactions between asperities, the displacement of each asperity is obtained by summing the displacements due to all the contacting asperities.

If $N$ is the number of the supposed contacting asperities, the displacement $w_{k}$ of the $k^{\text {th }}$ asperity reads

$$
N w k=\mathrm{X} G_{i}
$$

$\left(r_{i k}\right)$

where

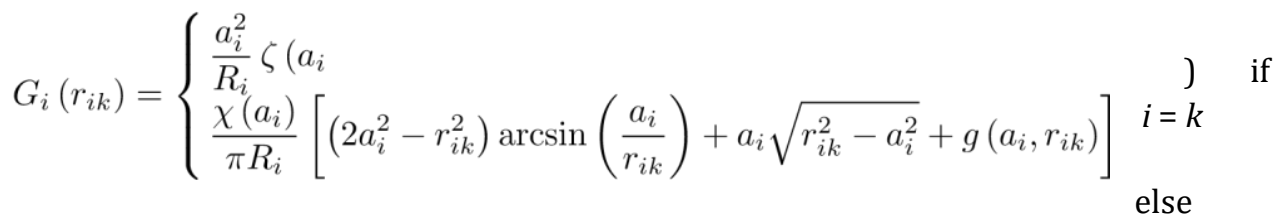

where $r_{i k}$ is the horizontal distance between the $k^{\text {th }}$ and the $i^{\text {th }}$ asperity. This expression has been obtained from the simplified asperity contact model developed in the last section (see 30 in Appendix C.) 
Let us denote $z_{M}$ the maximum height of the rough surface and $z_{i}$ the height of the $i^{t h}$ asperity, the prescribed displacement of the $i^{\text {th }}$ asperity is given by:

$$
\delta_{k}=\mathrm{h} \delta-\left(Z_{M}-Z_{k}\right) \mathrm{i}
$$

where $<$. $>$ is the positive part of its operand.

Solving the problem is to find a distribution of contact dimensions $\mathbf{a}=\left(a_{1}, a_{2}, \ldots, a_{N}\right)$ satisfying contact conditions: $w_{k} \geq \delta_{k}$.

The equality means that the asperity is in contact while the strict inequality signifies that the asperity is not in contact, and in this case, the change of the asperity height is due to interaction effects.

Unlike our previous work [11], where the problem has been solved using a direct method based on the minimization of an objective function based on contact kinematic conditions, the present problem is solved with a variational method consisting in minimizing of the total complementary potential energy $U_{E}$ of the solid [14]

$$
U_{E}=\frac{1}{2} \int_{\Gamma_{c}} p \cdot u_{z} d S-\int_{\Gamma_{c}} p \cdot \delta d S
$$

where $\Gamma_{c}$ is the contact zone, $p$ is the contact pressure field and $\delta$ is the prescribed displacement field.

For a given asperity, using the asperity contact model, the first integral corresponds to the internal energy and reads

$$
\begin{aligned}
\int_{\Gamma_{j}} p . u_{z} d S & =\frac{-8 \alpha \chi\left(a_{j}\right)}{R_{j}} \int_{\Gamma_{j}} \sqrt{a_{j}^{2}-r^{2}} \sum_{l}^{N} G_{l}\left(r_{l j}\right) r d r \\
& =\frac{-8 \alpha}{3} \frac{\chi\left(a_{j}\right) a_{j}^{3}}{R_{j}}\left(\sum_{l \neq j}^{N} G_{l}\left(r_{l j}\right)+\frac{a_{j}^{2}}{R_{j}}\left(\zeta\left(a_{j}\right)-\frac{1}{5}\right)\right)
\end{aligned}
$$

and the second integral is the prescribed displacements work which is given by

$$
\begin{aligned}
\int_{\Gamma_{j}} p . \delta d S & =\frac{-8 \alpha \chi\left(a_{j}\right)}{R_{j}} \int_{\Gamma_{j}} \sqrt{a_{j}^{2}-r^{2}}\left(\delta_{j}-\frac{r^{2}}{2 R_{j}}\right) r d r \\
& =\frac{-8 \alpha}{3} \frac{\chi\left(a_{j}\right) a_{j}^{3}}{R_{j}}\left(\delta_{j}-\frac{1}{5} \frac{a_{j}^{2}}{R_{j}}\right)
\end{aligned}
$$

By summing the contribution of all the contacting asperities, the total complementary energy reads

$$
U_{E}=\frac{-4 \alpha}{3} \sum_{j}^{N} \frac{\chi\left(a_{j}\right) a_{j}^{3}}{R_{j}}\left(\sum_{l \neq j}^{N} G_{l}\left(r_{l j}\right)+\frac{a_{j}^{2}}{R_{j}}\left(\zeta\left(a_{j}\right)+\frac{1}{5}\right)-2 \delta_{j}\right)
$$

For the special case of homogeneous half spaces $\chi=\zeta=1$, this energy reads

$$
U_{E}=\frac{-4 \alpha}{3} \sum_{j}^{N} \frac{a_{j}^{3}}{R_{j}}\left(\sum_{l \neq j}^{N} G_{l}\left(r_{l j}\right)+\frac{6}{5} \frac{a_{j}^{2}}{R_{j}}-2 \delta_{j}\right)
$$


The problem is solved by minimizing the system total energy under the constraints $a \geq 0$. A Newton likewise method is used to minimize this quantity [42].

Once the optimal contact radius distribution is found, the total force and the real contact area are obtained by adding the contribution of all the contacting asperities, hence they are respectively given by

$$
\begin{gathered}
P=-\frac{8}{3} \alpha \sum_{i}^{N} \chi\left(a_{i}\right) \frac{a_{i}^{3}}{R} \\
A=\pi \sum_{i}^{N} a_{i}^{2}
\end{gathered}
$$

The normal separation between the two faces is

where ${ }^{-} z$ is the height of the mean plane.

$$
g_{n}=\left(z_{M}-z^{-}\right)-\delta
$$

As stated before, this model considers the interactions between asperities. A simple form can be proposed to cover the case where interactions are not considered. Indeed, by neglecting the interaction terms $G_{j}$, the contact radius for each asperity can be computed from the following equation

$$
\delta_{i}=\frac{a_{i}^{2}}{R_{i}} \zeta\left(a_{i}\right)
$$

This formula can be obtained directly from contact kinematic conditions.

\section{Results}

As an example, the normal contact of a two-layered elastic solid with a rigid plan plane is investigated. The surface of the solid is rough (see Fig.14 (a)). For instance, surface roughness is kept fixed while the solid layer parameters are varied. For each configuration, the obtained results are compared to finite element calculations in the same manner as it was done for the single asperity problem (see section 3.2). Moreover, the effect of the solid parameters and interactions is discussed.

The dimensions of the surface sample are $1 \mathrm{~mm} \times 1 \mathrm{~mm}$. A fractal self-affine rough surface is considered (see Fig.14 (c)). Roughness is generated numerically using numerical tools based on the surface spectral density (PSD). This means that its power spectrum density (PSD) is approximately given in the form of a power law $P S D \sim|\mathbf{k}|^{-2\left(4-D_{f}\right)}$, where $D_{f}$ is the fractal dimension and $\mathbf{k}$ is the wave vector. In this paper, the fractal dimension of the surface is fixed at $D_{f}=2.4$. The selfaffinity is considered between two cut-off wave numbers $k_{m}$ and $k_{M}$. The discrete interval $\left[k_{m}, k_{M}\right]$ defines the breadth of the roughness spectrum and the PSD vanishes elsewhere. The cutoff wave numbers have been first fixed at $k_{m}=2$ and $k_{M}=10$, but will be varied later to discuss their effect. For the chosen values of the cutoff wave numbers, the studied surface corresponds to a narrow band of roughness. Using the PSD, the rough surface has been generated numerically 
using the discrete Fourier Transform [5]. In particular, the height profile $z$ is

generated by the equation $z(\mathbf{x})=z_{0} \sum P S D^{\frac{1}{2}}(\mathbf{k}) e^{i \cdot\left(\frac{2 \pi}{L} \mathbf{k} \cdot \mathbf{x}+\phi(\mathbf{k})\right)}$ where $z_{0}$ is a scaling amplitude

k parameter, $L$ is the root square of the surface area and $\varphi(\mathbf{k})$ are independent random variables which are uniformly distributed in the interval $[0,2 \pi[$. Once the random rough surface is generated, the asperities are identified as local maxima and their geometrical properties are computed using the finite difference method. The fractal dimension of the surface is 2.4 and the root mean square is $3.4 \mu \mathrm{m}$. The surface asperities are identified as local maxima. Their geometrical properties are computed using the finite difference method. In the finite element model (FEM model), illustrated in Fig.14, the solid is meshed with $6.10^{5}$ quadratic hexahedron elements (see Fig.14 (b)). At the top of the rough surface, there is $\sim 3.10^{4}$ elements. The convergence of the numerical models has been checked and the number of the considered elements is high enough to capture all surface details. The contact problem is solved using Augmented Lagrangian formulation [13] which guarantees the FEM solving efficiency. The finite element analysis is performed with Abaqus/Standard 6.13.

The results from various models are shown in Fig.15 and Fig.16. The first layer thickness is $h=100 \mu \mathrm{m}$, its Young modulus is fixed at $E_{1}=4 G P a$ and the ratio $E_{2} / E_{1}$ is varied from 0.2 to 5 .

The variation of the dimensionless contact pressure $p_{n} / E_{1}^{*}$ (where $E_{1}^{*}=-2 \alpha_{\text {is }}$ the equivalent elastic modulus of the first layer and $p_{n}$ is the mean contact pressure) with normal separation $g_{n}$ is shown in Fig.15. At first sight, there is a good accordance between the proposed model predictions and finite element results. One also can see that the impact of the sub-layer properties is considerable. It is also noticeable that the evolution between the logarithm of $p_{n} / E_{1}^{*}$ and $g_{n}$ is almost linear except for the case of small loadings, and this for the different tested configurations.

In Fig.16, the evolution of the real contact area fraction $A / A_{0}$ (where $A_{0}$ is the apparent contact area) with the quantity $p_{n} / E_{1}^{*}$ is presented. The numerical predictions are in good agreement with the model results. The contact area mean pressure relationship is almost linear except for the case of very small fractions of contact area $\left(A / A_{0} \leq 0.5 \%\right)$. However, the most interesting observation that can be made is that this evolution is marginally affected by the sub-layer modulus. Indeed, the first layer modulus is the same for all the considered configurations and the introduced function $\chi$ is equal to 1 (see 


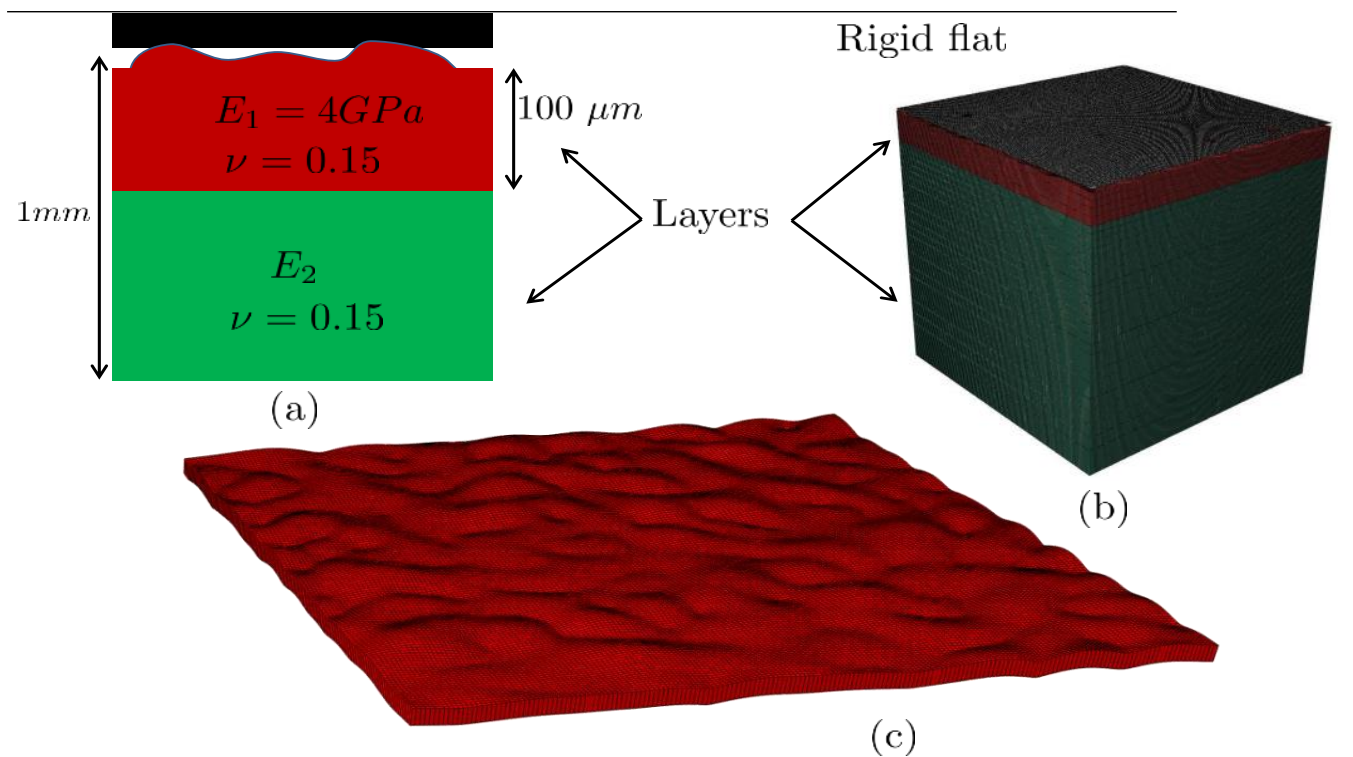

Fig. $14(a)$ Schematic of the model. The first layer thickness is $h=100 \mu m$ and the following parameters are considered : $H=1 \mathrm{~mm}$ and $E_{1}=4 G P a$. $(b)$ Schematic of the finite element model. The two-layered solid is meshed with $6.10^{5}$ hexahedron elements. (c) Illustration of surface roughness.

Figs.7-10), independently of the sub-layer modulus. Therefore, the pressure level which is controlled by function $\chi$ (see Eq.29), is kept fixed with respect to contact area. Thus, this evolution is mainly affected by roughness and the first layer modulus. Finally, one should expect that the linearity of the area vs pressure relationship is only valid for a limited range of contact area, as in the classical asperity theories [7, 9].

From these examples, it is clear that the elastic properties of the sub-layers have a considerable influence on the separation-load relation but marginally effect on the contact area-load evolution. It seems that this relationship depends only on the first layer modulus which was fixed in this study.

Another interesting point to be investigated is the interaction effect on the system response. Up to now, the complete model considering interactions has been tested and results are in good accordance with FEM calculations. In what follows, the results from the complete model (that is indicated as "I model ") are compared to the simplified model which does not consider interaction (indicated as "N model ").

The evolution of $p_{n} / E_{1}^{*}$ with normal separation $g_{n}$ is shown for both models in Fig.17. As we can see, the contribution of interactions depends on the solid parameters, and in this example, it is the ratio $E_{2} / E_{1}$. The increase of $E_{2} / E_{1}$ attenuates the effect of interactions. For higher values of this ratio, interactions has little effect on the studied curve. Indeed, the function $g$ introduced 


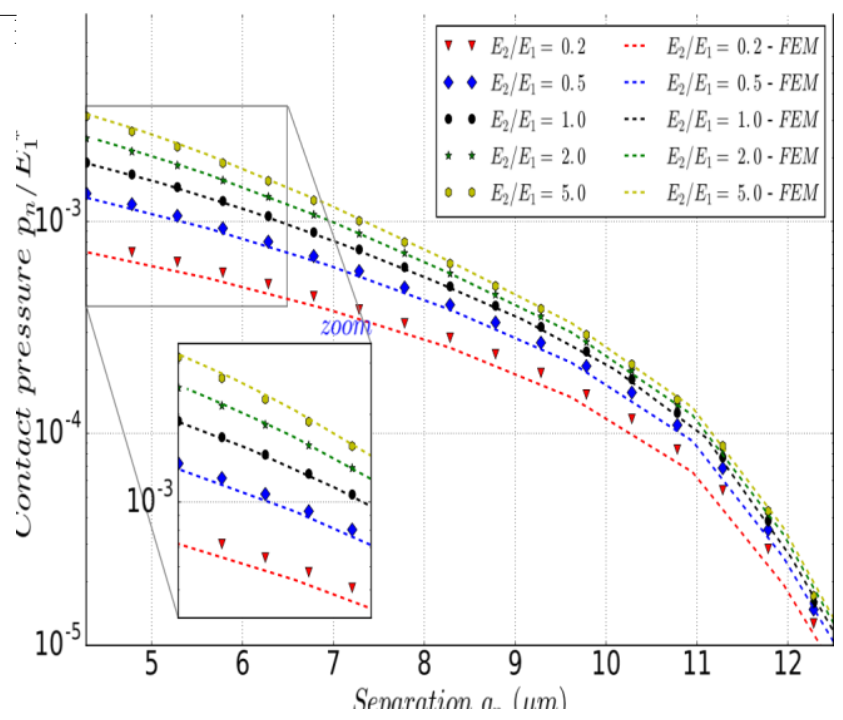

Fig. 15 Evolution of the dimensionless contact pressure with the normal separation. Comparison between FEM results and the model predictions.

in Eq.30 has a great effect on interaction terms. For instance, for the case where $E_{2} / E_{1}=5$, these terms almost vanish and there is no need to consider interactions. In contrast, if the sub-layer is less rigid than the surface layer $\left(E_{2} / E_{1} \leq 1\right)$, the non-consideration of asperities lead to an overestimation of the global stiffness.

Hence one can conclude that the major influence of the sub-layers properties on the interface behavior lies in the interactions between asperities, which affect subsequently the contact area and normal stiffness. It is also worth to notice that the influence of interactions is not only dependent on the sub-layers properties but also on the first layer thickness.

Regarding the evolution of the real contact area with loading (see Fig.18), we observe that interactions do not affect this evolution. This remark have been already made in many works studying the effect of interactions on loading-area evolution (see $[9,11])$.

With regard to the computational time, the new contact model provides results within ten to twenty minutes if interactions are included, and few seconds if not. The fully discretized solution obtained with the finite element method is more computationally expensive and lasts 6-8 hours using a high-performance computer. Thus the present model is numerically efficient. 


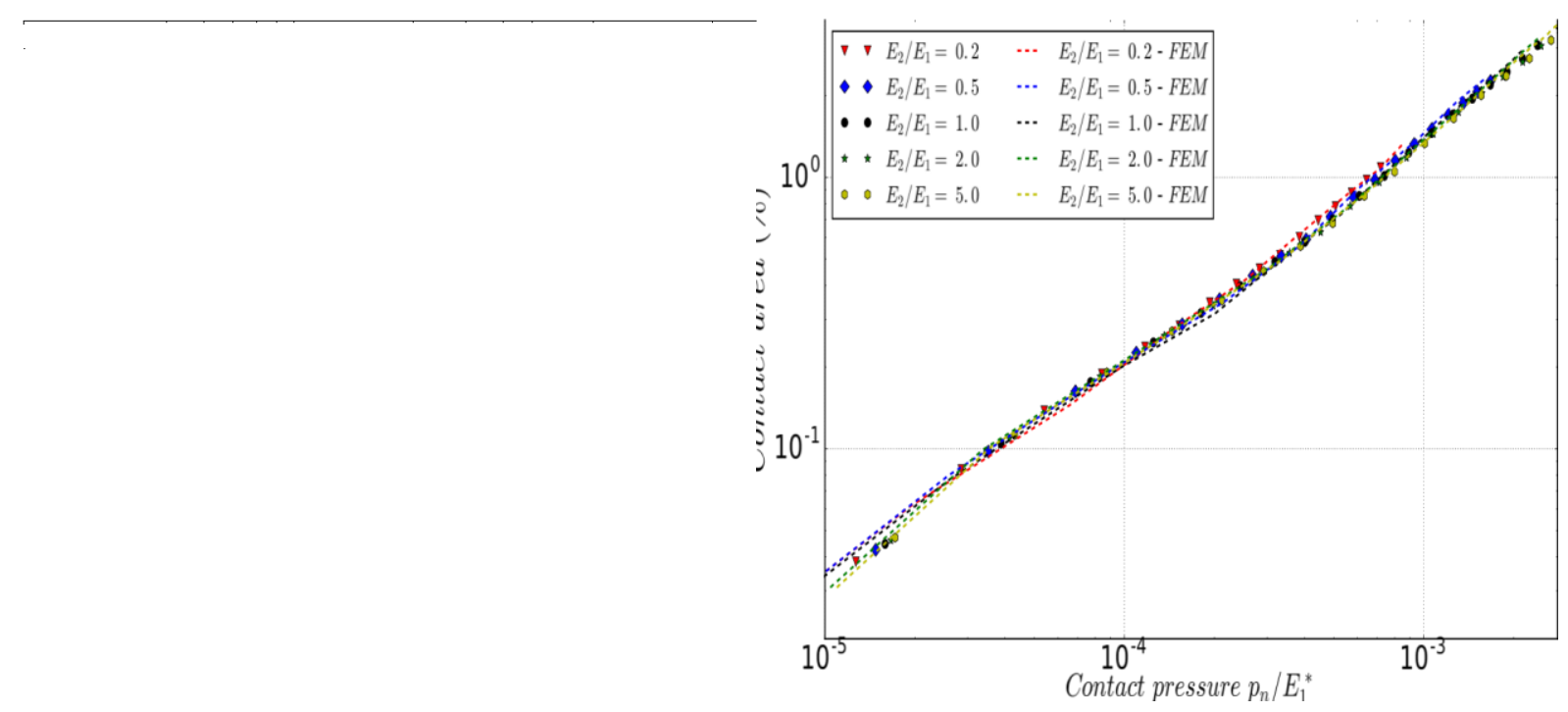

As is aforementioned, the precedent studies were conducted on a narrow band rough surface and the results have been verified with the finite element method. In what follows, we present the results obtained with this model using different Fig. 16 Evolution of the fraction of the contact real area with the dimensionless contact pressure. Comparison between FEM results and the model predictions.

kinds of roughness. Indeed, the upper cut-off wave number $k_{M}$ has been varied from 10 to 20 and then to 40 . For each value of $k_{M}, 20$ surface samples have been studied. The purpose is to investigate the dependency of the effects of interactions and the sub-layer parameters on the surface spectrum bandwidth. Moreover, from this study the deviation from one result to another can be assessed and the global tendencies can be analyzed if they exist.

In Fig. 19 is shown the evolution of the dimensionless contact pressure with the normal separation for the case where $E_{2} / E_{1}=1$ and considering the two models : "I model " if interactions are considered and " $\mathrm{N}$ model " if not. As can be seen from this figure, the spectrum breadth have a considerable influence on the predictions of the model if interactions are not considered (see the red curves in the same figure). However, interestingly the gap between the same curves decrease if interactions are considered especially for small separations (see the blue curves). For large separations, a considerable scatter is observable which can be due to the dependency of the results at this stage on the arrangement of asperities. Once again, this study confirms that interactions tend to reduce the slope of the pressure vs separation relationship despite the spectrum bandwidth value. As regards the contact area vs pressure curve (see Fig. 20), 


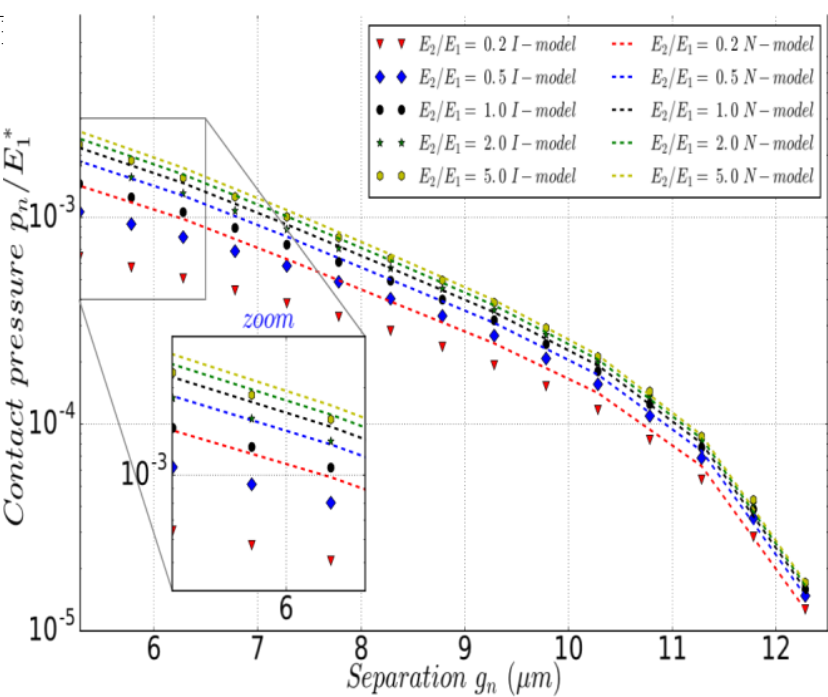

one can see that this curve depends considerably on the spectrum breadth. The contact area decreases with the increase of $k_{M}$. This behavior is predictable since the asperities become much smaller when $k_{M}$ increases. Interestingly, one can also remark that interactions do not modify these curves

Fig. 17 Evolution of the dimensionless contact pressure with the normal separation. Comparison between the complete model (" I model ") and the one which does not consider interactions (" N model")

and this for the different surface samples.

Similarly, we have conducted the same study considering $E_{2} / E_{1}=5$. The evolution of the pressure vs separation is shown in Fig.21. Comparing to the previous case, it is clear that interactions become less influent on this relationship especially for the narrow band surfaces $k_{M}=10$. As $k_{M}$ increases, interactions still have an impact on this relationship. This can also be explained by the fact that the increase of $k_{M}$ leads to decreasing the contact spots size with respect to the surface layer thickness which remains fixed at $100 \mu \mathrm{m}$. Moreover, for the same configurations, Fig.22 shows the area vs pressure curve. Once again, the same results can be seen, namely the considerable influence of $k_{M}$ and conversely the non-dependence on interactions.

From the different studies, it is clear that the prediction of the pressure vs separation curve depends on the spectrum bandwidth and the solids layers properties. As we have shown, the consideration of interactions is proved to be necessary for many cases, in particular, if the surface spectrum bandwidth is enough large and / if the surface layer is enough thick or if the solid sub-layers 
are less stiff. Moreover, if interactions are considered, the predictions of the model seems to be less dependent on the upper wave number especially for the case of small separations. Furthermore, the contact area vs pressure curve seems to be marginally affected by all these features, except the spectrum bandwidth, and the linearity is predicted for limited range of contact areas. In particular, the increase of the surface bandwidth leads to a decrease of the 


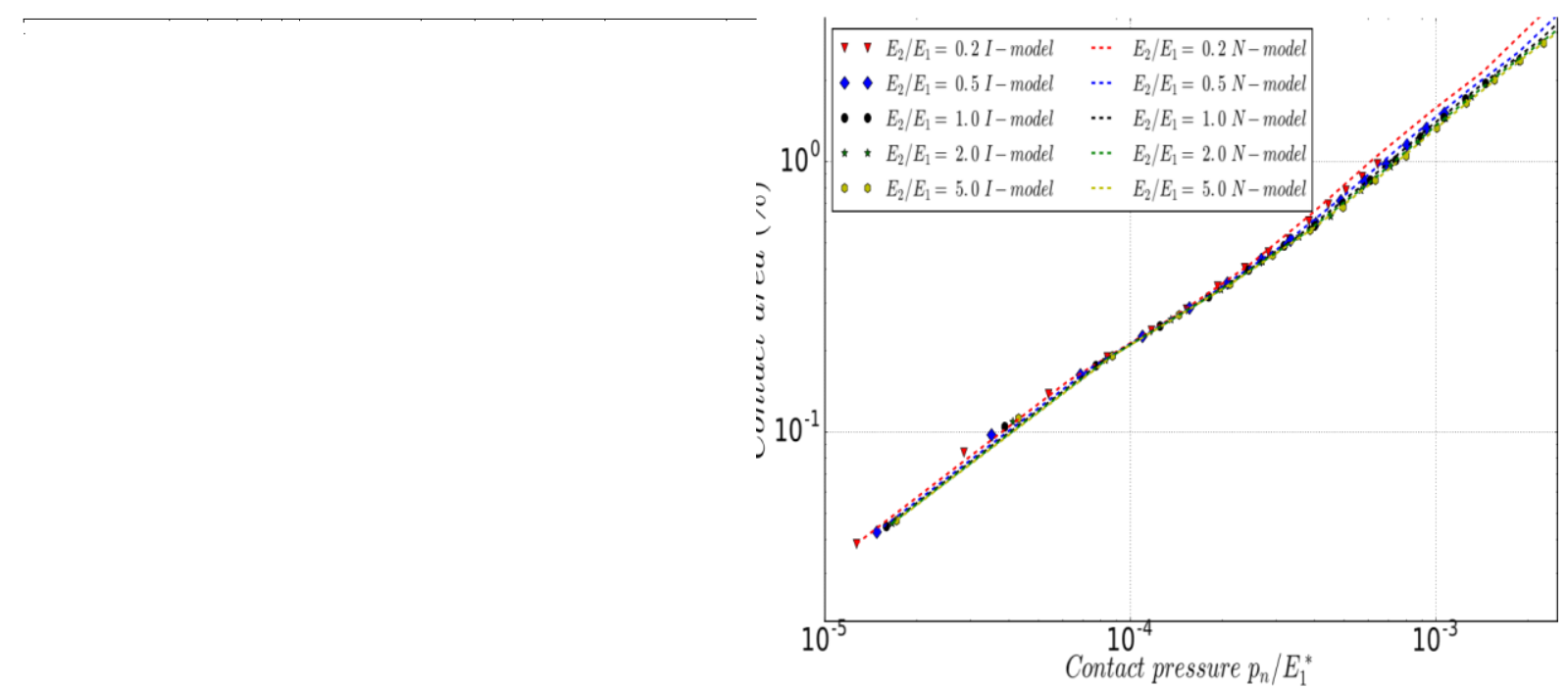

Fig. 18 Evolution of the fraction of the contact real area with the dimensionless contact pressure. Comparison between the complete model (" I model ") and the one which does not consider interactions (" $\mathrm{N}$ model ")

contact area with respect to the load. This is a classical result and was already predicted in many theories such as Bush et al. theory [8] and Persson's theory [43].

\section{Conclusion}

In this work, a contact model of rough surfaces and multi-layered solids has been proposed. The model is based on a multi-asperity surface description. The transfer matrix technique has been used to express surface displacements as functions of the surface loads. Then, making use of Abel and Hankel transforms, a contact model of parabolic asperities has been developed. At the asperity scale, results show that the sub-layer properties affect clearly the load-displacement curve.

By making use of a second order approximation of the asperity model, a contact model of rough surfaces has been developed. The obtained results have been compared to finite element calculations. A good agreement has been observed between the two methods.

As an example, a two-layered solid with a rough surface has been studied. Results show that the sub-layer properties strongly affect the contact interface 


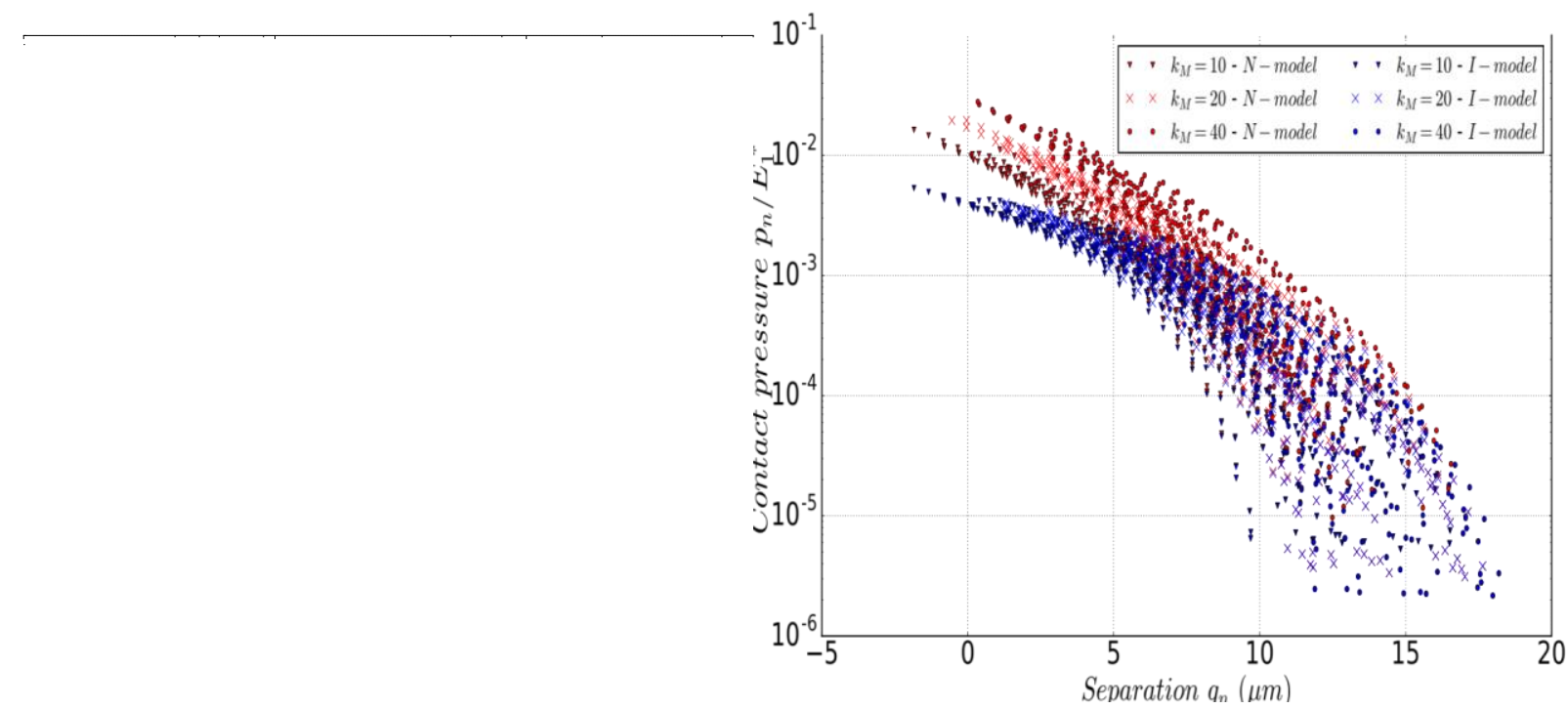

stiffness but much less the load-area evolution. The latter depends essentially on surface roughness and the first layer properties. With regard to stiffness,

Fig. 19 Evolution of the dimensionless contact pressure with the normal separation. Comparison between the complete model (" I model ") and the one which does not consider interactions (" N model ") for different values of the upper wave number $k_{M}(10,20$ and

$40), E_{2} / E_{1}=1$ and 20 surface samples

the sub-layers properties have a great influence on interaction terms which affects the load-separation evolution. Indeed, it has been shown that in some cases, the interactions between asperities can be neglected, while in the homogeneous case, interactions must be considered even in the case of small loads and contact areas. Moreover, the effect of the surface bandwidth has been investigated over many surface realizations. The obtained results highlight the role of interactions which increase if the surface is a large band while the contact area decreases since the scale of asperities decreases with the upper wave vector increasing.

Finally, this model can be used to embed large scale numerical models with the aim to consider the effect of roughness and a normal gradient of properties using the approach presented in our work [11].

\section{Acknowledgments}

The present research work has been supported by the International Campus on Safety and Intermodality in Transportation, the Hauts-de-France Region, the 


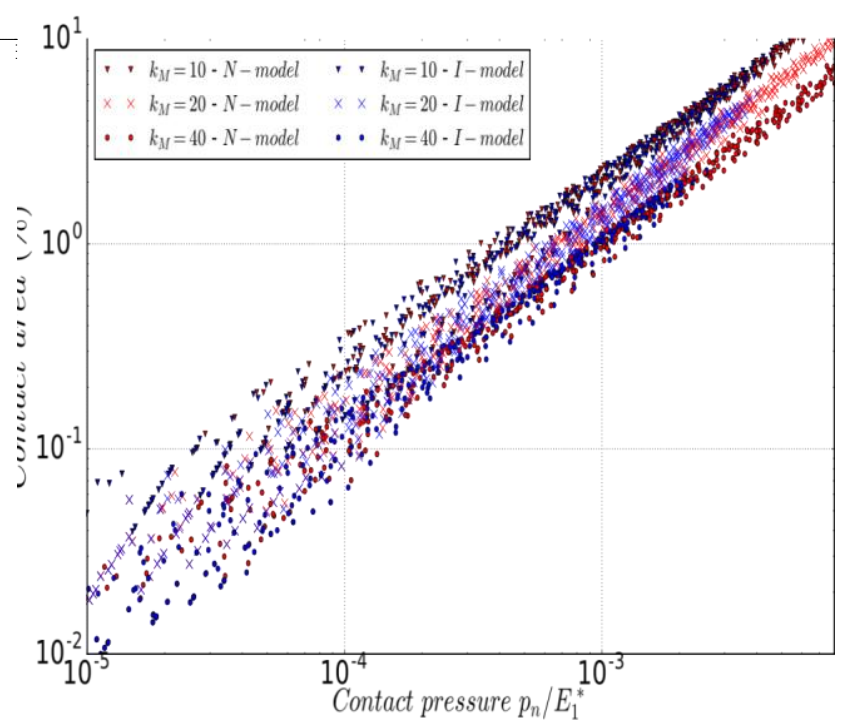

European Union, the Regional Delegation for Research and Technology, the Ministry of Higher Education and Research, the French National Research Agency (ANR COMATCO), and the National Center for Scientific Research. The authors gratefully acknowledge these institutions for their support.

Fig. 20 Evolution of the fraction of the contact real area with the dimensionless contact pressure. Comparison between the complete model (" I model ") and the one which does not consider interactions (" $\mathrm{N}$ model ") for different values of the upper wave number $k_{M}(10,20$ and 40$), E_{2} / E_{1}=$ 1 and 20 surface samples

\section{References}

[1] Hetzler, H. \& Willner, K. On the influence of contact tribology on brake squeal. Wear, 2012, 46, 237-246

[2] Longuet-Higgins, M. S. The statistical analysis of a random, moving surface. Philosophical Transactions of the Royal Society of London. Series A, Mathematical and Physical Sciences, 1957, 321-387

[3] Nayak, P. R. Random process model of rough surfaces. Journal of Tribology, American Society of Mechanical Engineers, 1971, 93, 398-407

[4] Majumdar, A., \& Tien, C. L. Fractal characterization and simulation of rough surfaces. Wear, 1990, 136(2), 313-327

[5] Persson, B., Albohr, O., Tartaglino, U., Volokitin, A. \& Tosatti, E. On the nature of surface roughness with application to contact mechanics, sealing, rubber friction and adhesion. Journal of Physics: Condensed Matter, IOP Publishing, 2005, 17, R1-R62

[6] Archard, J. F. Elastic deformation and the laws of friction. Proceedings of the Royal Society of London. Series A. Mathematical, Physical and Engineering Sciences, The Royal Society, 1957, 243, 190-205

[7] Greenwood, J. \& Williamson, J. Contact of nominally flat surfaces. Proceedings of the Royal Society of London. Series A. Mathematical and Physical Sciences, The Royal Society, 1966, 295, 300-319 


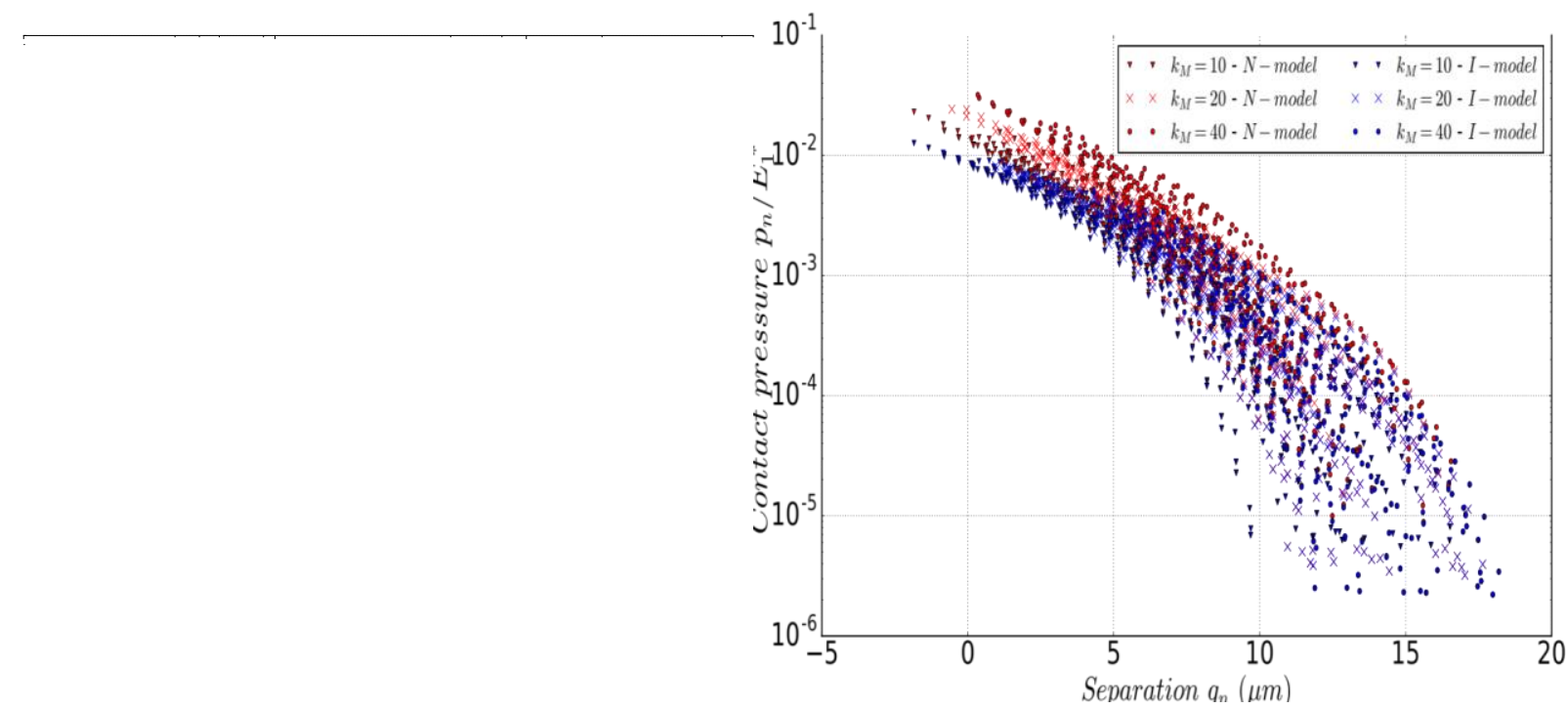

[8] Bush, A., Gibson, R. \& Thomas, T. The elastic contact of a rough surface. Wear, 1975, 35, 87-111

[9] Ciavarella, M., Delfine, V. \& Demelio, G. A re-vitalized Greenwood and Williamson model of elastic contact between fractal surfaces. Journal of the Mechanics and Physics of Solids, 2006, 54, 2569-2591

[10] Saha, S., Xu, Y., \& Jackson, R. L. Perfectly elastic axisymmetric sinusoidal surface asperity contact. Journal of Tribology, 2016, 138(3), 031401.

Fig. 21 Evolution of the dimensionless contact pressure with the normal separation. Comparison between the complete model (" I model ") and the one which does not consider interactions (" N model ") for different values of the upper wave number $k_{M}(10,20$ and 40$), E_{2} / E_{1}=5$ and 20 surface samples

[11] Waddad, Y. , Magnier, V. , Dufr'enoy, P. \& De Saxc'e, G. A multiscale method for frictionless contact mechanics of rough surfaces. Tribol. int., 2016, 96, 109-121.

[12] Pei, L., Hyun, S., Molinari, J. F. \& Robbins, M. O.Finite element modeling of elastoplastic contact between rough surfaces. Journal of the Mechanics and Physics of Solids, 2005, 53, 2385-2409

[13] Wriggers, P. Computational contact mechanics. 2nd ed., Springer, 2006.

[14] Johnson, K. L. Contact mechanics. Cambridge university press, 1987

[15] Willner, K. Fully coupled frictional contact using elastic halfspace theory. Journal of Tribology, American Society of Mechanical Engineers, 2008, 031405, 1-8.

[16] Gallego, L., Nelias, D. \& Deyber, S. A fast and efficient contact algorithm for fretting problems applied to fretting modes I, II and III. Wear, 2010, 268, 208-222.

[17] Wang, Z., Jin, X., Liu, S., Keer, L. M., Cao, J., \& Wang, Q. A new fast method for solving contact plasticity and its application in analyzing elasto-plastic partial slip. Mechanics of Materials, 2013, 60, 18-35.

[18] Kogut, L., \& Etsion, I. Elastic-plastic contact analysis of a sphere and a rigid flat. Journal of applied Mechanics, 2002, 69(5), 657-662

[19] Jackson, R. L., \& Green, I. A statistical model of elasto-plastic asperity contact between rough surfaces. Tribology International, 2006, 39(9), 906-914 


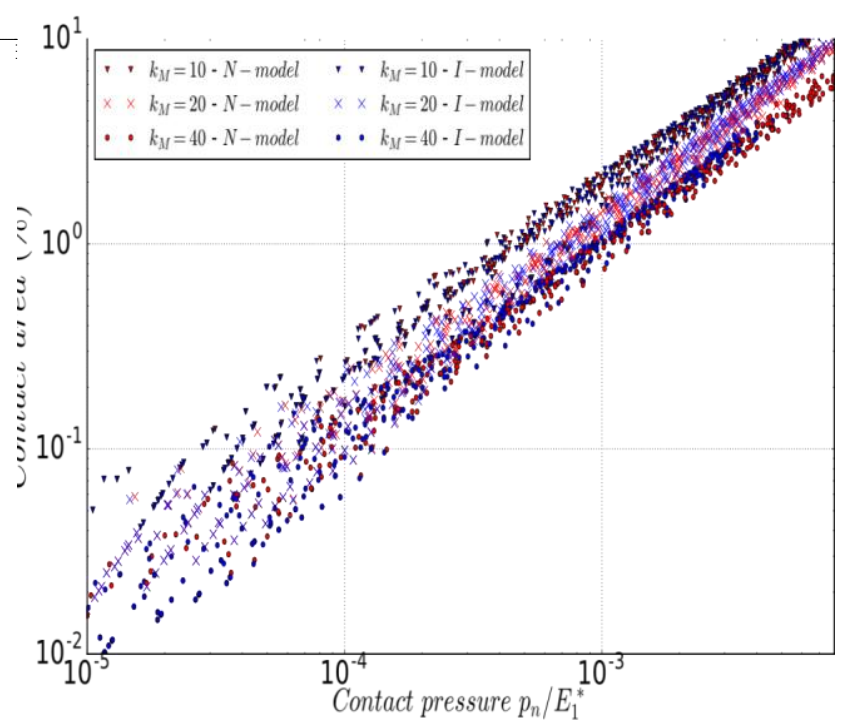

[20] Leroux, J., Fulleringer, B. \& Nelias, D. Contact analysis in presence of spherical inhomogeneities within a half-space. International Journal of Solids and Structures, 2010, 47, 3034-3049.

[21] Plumet, S. \& Dubourg, M. C. A 3-D model for a multilayered body loaded normally and tangentially against a rigid body: Application to specific coatings. Journal of tribology, 1998, 120(4), 668-676.

[22] Cole, S. J., \& Sayles, R. S. A numerical model for the contact of layered elastic bodies with real rough surfaces. Journal of tribology, 1992, 114(2), 334-340.

Fig. 22 Evolution of the fraction of the contact real area with the dimensionless contact pressure Comparison between the complete model (" I model ") and the one which does not consider interactions (" $\mathrm{N}$ model ") for different values of the upper wave number $k_{M}(10,20$ and 40$), E_{2} / E_{1}=$ 5 and 20 surface samples

[23] Mao, K., Bell, T., Sun, Y., \& Sayles, R. S. Effect of sliding friction on contact stresses for multilayered elastic bodies with rough surfaces. Journal of tribology, 1997, 119(3), 476-480.

[24] Liu, S. B., Peyronnel, A., Wang, Q. J., \& Keer, L. M. An extension of the Hertz theory for threedimensional coated bodies. Tribol. Lett., 2005, 18(3), 303-314.

[25] Nogi, T., \& Kato, T. Influence of a hard surface layer on the limit of elastic contactPart I: Analysis using a real surface model. Journal of Tribology, 1997, 119(3), 493-500.

[26] O'sullivan, T. C., \& King, R. B. Sliding contact stress field due to a spherical indenter on a layered elastic half-space. Journal of Tribology, 1988, 110(2), 235-240.

[27] Pasaribu, H. R., \& Dirk J. Schipper. Application of a deterministic contact model to analyze the contact of a rough surface against a flat layered surface. Journal of Tribology, 2005, 127(2), 451-455.

[28] Huajian, G., Cheng-Hsin, C., \& Jin, L. Elastic contact versus indentation modeling of multi-layered materials. International journal of Solids and Structures, 1992, 29(20), 2471-2492.

[29] Cai, Shaobiao \& Bhushan, Bharat A numerical three-dimensional contact model for rough, multilayered elastic/plastic solid surfaces. Wear, 2005, 259, 1408-1423.

[30] Peng, Wei \& Bhushan, Bharat Three-dimensional contact analysis of layered elastic/plastic solids with rough surfaces. Wear, 2001, 249, 741-760. 
[31] Kral, E. R., \& K. Komvopoulos.Three-dimensional finite element analysis of subsurface stress and strain fields due to sliding contact on an elastic-plastic layered medium. Journal of Tribology, 1997, 119(2), 332-341.

[32] Komvopoulos, K. \& Ye. N. Three-dimensional contact analysis of elastic-plastic layered media with fractal surface topographies. TRANSACTIONS-AMERICAN SOCIETY OF MECHANICAL ENGINEERS JOURNAL OF TRIBOLOGY, 2001, 123(3), 632-640.

[33] Goltsberg, R., I. Etsion, \& G. Davidi. The onset of plastic yielding in a coated sphere compressed by a rigid flat. Wear, 2011, 271(11), 2968-2977. 
[34] Song, W., Li, L., Ovcharenko, A., Jia, D., Etsion, I., \& Talke, F. E. Plastic yield inception of an indented coated flat and comparison with a flattened coated sphere. Tribology International, 2012, 53, 61-67.

[35] Singh, Sarva Jit Static deformation of a transversely isotropic multilayered half-space by surface loads. Physics of the earth and Planetary Interiors, 1986, 4, 263-273.

[36] Ernian, Pan Static response of a transversely isotropic and layered half-space to general surface loads. Physics of the Earth and Planetary Interiors, 1989, 54, 353-363.

[37] Yue, Z. Q. Elastic field for eccentrically loaded rigid plate on multilayered solids. International Journal of Solids and Structures, 1996, 33, 4019-4049. [38] Sneddon, I. N. Fourier transforms. Courier Corporation, 1995.

[39] Sneddon, I. N. The relation between load and penetration in the axisymmetric boussinesq problem for a punch of arbitrary profile. International Journal of Engineering Science, 1965, 3(1):47-57.

[40] Yu, H. Y., Sanday, S. C., \& Rath, B. B. The effect of substrate on the elastic properties of films determined by the indentation testaxisymmetric boussinesq problem. Journal of the Mechanics and Physics of Solids, 1990, 38(6), 745-764.

[41] Constantinescu, A., Korsunsky, A. M., Pison, O., \& Oueslati, A. Symbolic and numerical solution of the axisymmetric indentation problem for a multilayered elastic coating. International Journal of Solids and Structures, 2013, 50(18), 2798-2807.

[42] Nash, S. G. A survey of truncated-Newton methods. Journal of Computational and Applied Mathematics, 2000, 124, 45-59

[43] Persson, B., Bucher, F. \& Chiaia, B. Elastic contact between randomly rough surfaces: comparison of theory with numerical results. Physical Review-Series B-, American Physical Society, $2002,65,184106$

\section{Appendix A}

- Elastic matrices

$$
\mathbf{Z}^{1}=\exp (\rho z) \cdot\left[\begin{array}{cc}
1 & \exp (-2 \rho z) \\
\mu \rho-\mu \rho \exp (-2 \rho z)
\end{array}\right]
$$

and

$$
\begin{aligned}
& {\left[\frac{1}{2 \mu} \exp (\rho z) \frac{1}{2 \mu} \exp (-\rho z) \frac{1}{2 \mu}\left(z-\frac{2(1-\nu)}{\rho}\right) \exp (\rho z) \frac{1}{2 \mu}\left(z+\frac{2(1-\nu)}{\rho}\right) \exp (-\rho z)\right.} \\
& 2=\frac{1}{2 \mu} \exp (\rho z)-\frac{1}{2 \mu} \exp (-\rho z) \frac{1}{2 \mu}\left(z+\frac{(1-2 \nu)}{\rho}\right) \exp (\rho z) \frac{1}{2 \mu}\left(-z+\frac{(1-2 \nu)}{\rho}\right) \exp (-\rho z) \\
& \exp (\rho z) \quad-\exp (-\rho z) \quad\left(z-\frac{1}{\rho}\right) \exp (\rho z) \quad\left(-z-\frac{1}{\rho}\right) \exp (-\rho z) \\
& \exp (\rho z) \quad \exp (-\rho z) \quad z \exp (\rho z) \quad z \exp (-\rho z)
\end{aligned}
$$

and $\exp 0$ is the exponential function.

\section{- Transfer matrices of a layer}

$$
\eta=0.5 \exp (\rho h) \cdot\left[\begin{array}{cc}
1+\exp (-2 \rho h) & -\frac{1}{\mu}(1-\exp (-2 \rho h)) \\
-\mu(1-\exp (-2 \rho h)) & 1+\exp (-2 \rho h)
\end{array}\right]
$$


and

$$
z \xi=\frac{\mu}{1-\nu}\left(\mathbf{T}_{1} \cosh (\rho h)+\mathbf{T}_{2} \sinh (\rho h\right.
$$

$\mathbf{T}$

where

$$
1=\left[\begin{array}{cccc}
\frac{1-\nu}{\mu} & -\frac{\rho h}{2 \mu} & \frac{\rho h}{4 \mu^{2}} & 0 \\
\frac{\rho h}{2 \mu} & \frac{1-\nu}{\mu} & 0 & -\frac{\rho h}{4 \mu^{2}} \\
\rho h & 0 & \frac{1-\nu}{\mu} & -\frac{\rho h}{2 \mu} \\
0 & -\rho h & \frac{\rho h}{2 \mu} & \frac{1-\nu}{\mu}
\end{array}\right]_{\& \mathbf{T}}^{\mathbf{T}} 2=\left[\begin{array}{cccc}
-\frac{\rho h}{2 \mu} & \frac{1-2 \nu}{\mu} & -\frac{3-4 \nu}{4 \mu^{2}} & \frac{\rho h}{4 \mu^{2}} \\
\frac{\rho h}{2 \mu} & \frac{\rho h}{2 \mu} & -\frac{\rho h}{4 \mu^{2}} & -\frac{1-\nu}{2 \mu^{2}} \\
-1 & \rho h & -\frac{\rho h}{2 \mu} & -\frac{\rho h}{2 \mu} \\
-\rho h & -1 & -\frac{1-2 \nu}{\mu} & \frac{\rho h}{2 \mu}
\end{array}\right]
$$

and $\cosh (0$ and $\sinh (0$ are the hyperbolic sine and cosine functions.

\section{Appendix B}

The transfer matrix that relates surface displacements to surface loads can be expressed as follows

$$
\mathbf{F}\left[\begin{array}{ccc}
F_{11} & F_{12} & 0 \\
F_{21} & F_{22} & 0 \\
0 & 0 & F_{33}
\end{array}\right]
$$

The matrix coefficients are obtained from boundary conditions. Two cases are examined here :

\section{- Case of an elastic half space}

The multi-layered solid lies on an elastic half space. Thus, the half space parameters $A_{\eta}^{(N+1)}, A_{z}^{(N+1)}$ and $B_{z}^{(N+1)}$ vanish. It follows that

$$
\left[\begin{array}{ll}
F_{11} & F_{12} \\
F_{21} & F_{22}
\end{array}\right]=\frac{1}{N_{44} N_{32}-N_{34} N_{42}}\left[\begin{array}{ll}
N_{12} & N_{14} \\
N_{22} & N_{24}
\end{array}\right]\left[\begin{array}{cc}
N_{44} & -N_{34} \\
-N_{42} & N_{32}
\end{array}\right]
$$

and

$$
F_{33}=\frac{M_{12}}{M_{22}}
$$

where $\mathbf{N}=\mathbf{S} \cdot \mathbf{Z}_{2}^{(N+1)}\left(z_{N}\right)$ and $\mathbf{M}=\mathbf{R} \cdot \mathbf{Z}_{1}^{(N+1)}\left(z_{N}\right)$ and $z_{N}$ is the total height of the solid.

\section{- Case of a rigid half space}

If the multi-layered solid lies on a rigid half space, the displacements $w_{\eta}^{(N)}, w_{\xi}^{(N)}$ and $w_{z}^{(N)}$ are set to be zero. One can deduce that 
and $\quad\left[\begin{array}{ll}F_{11} & F_{12} \\ F_{21} & F_{22}\end{array}\right]=\frac{1}{S_{44} S_{33}-S_{34} S_{43}}\left[\begin{array}{ll}S_{13} & S_{14} \\ S_{23} & S_{24}\end{array}\right]\left[\begin{array}{cc}S_{44} & -S_{34} \\ -S_{43} & S_{33}\end{array}\right]$

$$
F_{33}=\frac{R_{12}}{R_{22}}
$$

Appendix C

Using the closed form of $\varphi_{a}$ and the parameters $\chi$ and $\zeta$, the following approximated expressions can be deduced :

- The total normal force $P$

$$
P=-\frac{8}{3} \alpha \chi(a) \frac{a^{3}}{R}
$$

- The normal stress $\sigma_{z z}$

$$
\sigma_{z z}(r)=\frac{4 \alpha}{\pi R} \chi(a) \sqrt{a^{2}-r^{2}} \text { if } r \leq a
$$

- The normal displacement $u_{z}$ reads

$$
\begin{aligned}
& u(r)=\left\{\begin{array}{c}
\frac{1}{2 R}\left(2 a^{2} \zeta(a)-r^{2}\right) \\
(
\end{array}\right. \\
& \text { ( } \frac{\chi(a)}{\pi R}\left[\left(2 a^{2}-r^{2}\right) \arcsin \left(\frac{a}{r}\right)+a \sqrt{r^{2}-a^{2}}+g(a, r)\right] \text { else. }
\end{aligned}
$$$$
\text { where } g(a, r)=4 \int_{0}^{\infty} k_{z}(\rho) J_{0}(\rho r) \frac{\sin (a \rho)-a \rho \cos (a \rho)}{\rho^{3}} \mathrm{~d} \rho
$$ 